\title{
Accurate Appearance Preserving Prefiltering for Rendering Displacement-Mapped Surfaces
}

\author{
LIFAN WU, University of California, San Diego \\ SHUANG ZHAO, University of California, Irvine \\ LING-QI YAN, University of California, Santa Barbara \\ RAVI RAMAMOORTHI, University of California, San Diego
}

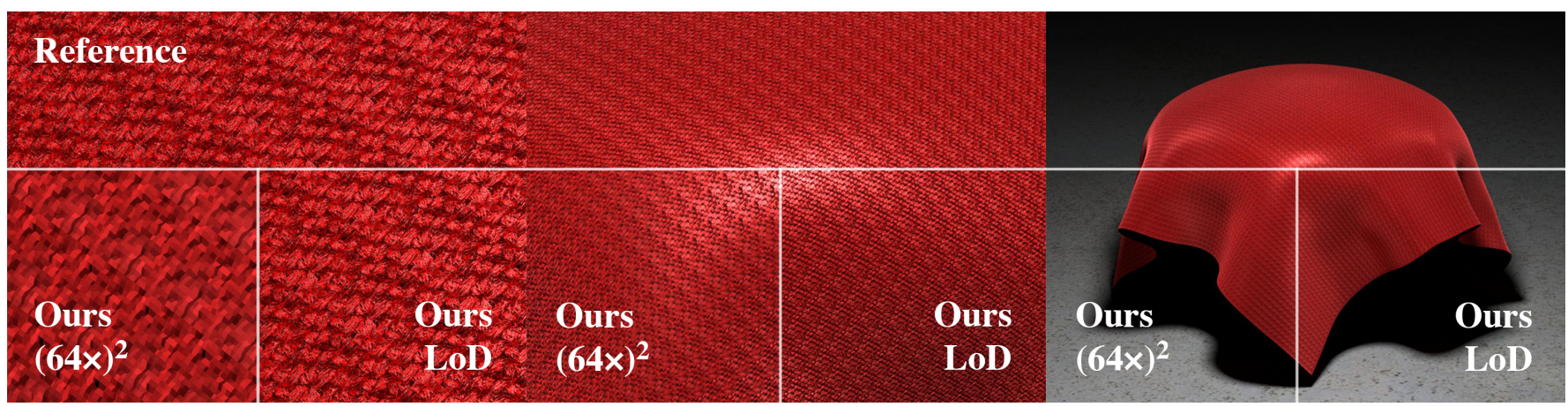

Fig. 1. We present a new approach to prefilter high-resolution displacement maps while preserving the input appearance. High-resolution displacement maps can produce rich geometric details (top-left) but they are difficult to prefilter. Our prefiltered model handles the change of shadowing, masking and interreflections caused by downsampling the displacement map. At a single scale, although the detailed micro-structures are different (see ours (64×) ${ }^{2}$ at left), our prefiltered model preserves the original appearance accurately when we view the object from a distance (see the right image). We can also combine our models at multiple downsampling scales to form a mipmap, enabling accurate and anti-aliased LoD rendering.

Prefiltering the reflectance of a displacement-mapped surface while preserving its overall appearance is challenging, as smoothing a displacement map causes complex changes of illumination effects such as shadowing-masking and interreflection. In this paper, we introduce a new method that prefilters displacement maps and BRDFs jointly and constructs SVBRDFs at reduced resolutions. These SVBRDFs preserve the appearance of the input models by capturing both shadowing-masking and interreflection effects. To express our appearance-preserving SVBRDFs efficiently, we leverage a new representation that involves spatially varying NDFs and a novel scaling function that accurately captures micro-scale changes of shadowing, masking, and interreflection effects. Further, we show that the 6D scaling function can be factorized into a $2 \mathrm{D}$ function of surface location and a $4 \mathrm{D}$ function of direction. By exploiting the smoothness of these functions, we develop a simple and efficient factorization method that does not require computing the full scaling function. The resulting functions can be represented at low resolutions (e.g., $4^{2}$ for the spatial function and $15^{4}$ for the angular function), leading to minimal additional storage. Our method generalizes well to different types of geometries beyond Gaussian surfaces. Models prefiltered using

Authors' addresses: Lifan Wu, University of California, San Diego, liw086@eng.ucsd edu; Shuang Zhao, University of California, Irvine, shz@ics.uci.edu; Ling-Qi Yan, University of California, Santa Barbara, lingqi@cs.ucsb.edu; Ravi Ramamoorthi, University of California, San Diego, ravir@cs.ucsd.edu.

Permission to make digital or hard copies of all or part of this work for personal or classroom use is granted without fee provided that copies are not made or distributed for profit or commercial advantage and that copies bear this notice and the full citation on the first page. Copyrights for components of this work owned by others than the author(s) must be honored. Abstracting with credit is permitted. To copy otherwise, or republish, to post on servers or to redistribute to lists, requires prior specific permission and/or a fee. Request permissions from permissions@acm.org.

(C) 2019 Copyright held by the owner/author(s). Publication rights licensed to ACM.

0730-0301/2019/7-ART137 \$15.00

https://doi.org/10.1145/3306346.3322936 our approach at different scales can be combined to form mipmaps, allowing accurate and anti-aliased level-of-detail (LoD) rendering.

CCS Concepts: • Computing methodologies $\rightarrow$ Rendering.

Additional Key Words and Phrases: multi-resolution, level of detail, prefiltering, global illumination

\section{ACM Reference Format:}

Lifan Wu, Shuang Zhao, Ling-Qi Yan, and Ravi Ramamoorthi. 2019. Accurate Appearance Preserving Prefiltering for Rendering Displacement-Mapped Surfaces. ACM Trans. Graph. 38, 4, Article 137 (July 2019), 14 pages. https: //doi.org/10.1145/3306346.3322936

\section{INTRODUCTION}

High-resolution displacement maps are commonly used to describe detailed micro-geometries that can produce richly diverse appearances. Compared to normal mapping, displacement mapping is more physically consistent and can offer more realistic self-shadowing and silhouettes. However, such realism comes at the cost of difficult prefiltering: smoothing a displacement map usually weakens its intrinsic shadowing and results in brightened overall appearance. Therefore, rendering a high-resolution displacement map without introducing severe aliasing generally requires significant supersampling, which is computationally expensive.

Previous displacement mapping techniques such as LEAN [Olano and Baker 2010] and LEADR [Dupuy et al. 2013] can produce antialiased renderings of rough surfaces. However, they assume the normals of the input surfaces to have Beckmann distributions, which is usually violated in practice and fundamentally limits the accuracy 


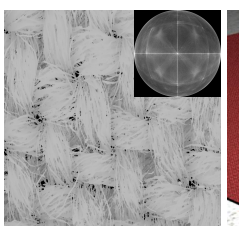

(a) Disp. map

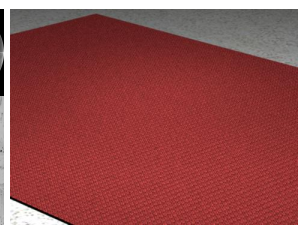

(b) Reference

$1000 \mathrm{spp}, 500 \mathrm{~s}$

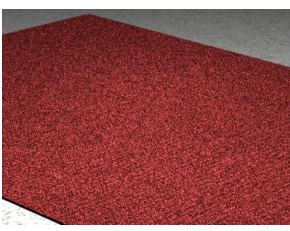

(c) Original

50 spp, 26s

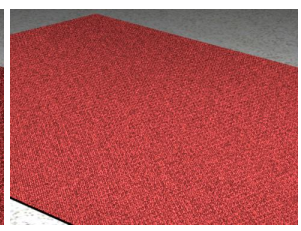

(d) Naïve

$80 \mathrm{spp}, 27 \mathrm{~s}$

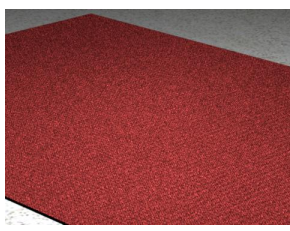

(e) Ours

35 spp, 26s

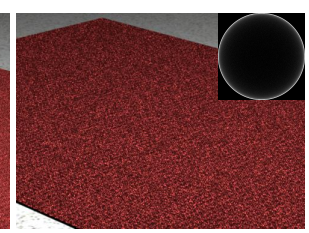

(f) LEADR

$70 \mathrm{spp}, 26 \mathrm{~s}$

Fig. 2. Equal-time renderings with only direct illumination. Given a high-resolution displacement map (a) and a highly glossy base BRDF as input, directly rendering using them results in aliased results (c). Simply downsampling the displacement map to a lower resolution but keeping the original base BRDF leads to inaccurate appearance (d). Our method (e) prefilters the displacement map and the base BRDF while preserving the input appearance (b), which produces accurate and anti-aliased renderings. Using the microfacet BRDF proposed in LEADR [Dupuy et al. 2013] generates inaccurate appearance (f) since the Gaussian surface assumption is violated. We show the ground truth NDF visualization of this displacement map in the top-right inset of (a), and the NDF estimated from LEADR in the top-right inset of (f), which is very different.

of these methods (Figure 2(f)). To handle a wider range of surfaces, some bi-scale appearance models [Wu et al. 2011; Iwasaki et al 2012] precompute the overall surface reflectance by averaging light reflections at the micro-scale. This yields one spatially macro-scale effective BRDF with no spatial variation, which has difficulties in reproducing micro-scale details at close-up views. Both approaches neglect interreflection and can lead to significant energy loss.

In this paper, we introduce a novel method to prefilter displacementmapped (opaque) surfaces while accurately preserving their overall appearances under both direct and global illuminations (e.g., Figures 1, 2). Given a high-resolution displacement map and an isotropic base micro-BRDF, we seek displacement maps and accompanying surface reflectance models that have reduced resolutions and closely preserve the macro-scale appearance of the input model. To this end, we leverage a generalized version of the effective BRDF formulation [Wu et al. 2011] that takes both shadowing-masking and interreflection effects into account. Based on this formulation, we directly match the effective BRDFs of the input model and our prefiltered variants.

Our method (Figure 3) starts with downsampling the input displacement map. This is achieved via an optimization that aims to preserve the surface's meso-scale geometries (e.g., normals) and minimize potential energy loss. The resulting surface, when coupled with the input reflectance model, generally leads to a different appearance (see Figure 2(d) for an example) due to the change of shadowing structures, distribution of normals, and interreflections caused by the downsampling. The main focus of this paper, therefore, is to find proper surface reflectance models (i.e., BRDFs) so that, when coupled with the downsampled geometries, they closely resemble the appearance of the input.

To fully preserve the detailed appearance of the input model using the downsampled geometry, a 6D spatially varying effective $\mathrm{BRDF}$ is generally required. Unfortunately, explicitly expressing this SVBRDF requires significant computation and storage, negating the benefits of displacement map downsampling. Instead, we decompose the $6 \mathrm{D}$ function into a $4 \mathrm{D}$ spatially varying normal distribution function (SVNDF) [Han et al. 2007] and a novel scaling function. The SVNDF, which neglects both shadowing-masking and interreflection, primarily encodes spatial variations of the effective BRDFs and can be represented as $2 \mathrm{D}$ spatially varying parametric distributions. The scaling function, in contrast, accounts for the shadowing-masking and interreflection effects and is vital for matching the input appearance. To efficiently describe this scaling function, which is $6 \mathrm{D}$ itself, we factorize it into a $2 \mathrm{D}$ spatial scaling function of surface location and a $4 \mathrm{D}$ angular scaling function of direction (e.g., Figure 10). Further, we exploit the fact that the scaling functions are usually low-frequency and smooth in practice to introduce a simple and efficient factorization method to compute them from a sparse set of samples. With a resolution up to $4^{2}$ for the spatial function and $15^{4}$ for the angular one, our scaling functions only consume $200-400 \mathrm{~KB}$ of storage, making our prefiltered models compact and practically useful. Our contributions include:

- We develop a novel method to prefilter the surface reflectance of a high-resolution displacement map while accurately preserving the overall appearance (Figures 1, 16, 17, 18). This is achieved by first downsampling the displacement map to minimize the meso-scale average surface slopes ( $\$ 4.1)$, then separating a 6D SVBRDF into a SVNDF (§4.2) and a scaling function (§4.3).

- To match the target appearance, we utilize two scaling functions. One captures only shadowing-masking ( $\$ 4.3)$ and the other effectively handles both shadowing-masking and interreflection (§5.2). To model interreflection within the micro-geometry, which is generally missing in previous work, we introduce a generalized formulation of effective BRDFs (§5.1).

- We show the scaling function can be factorized as the product of a single spatial scaling function and a single angular scaling function. Exploiting their low-frequency property and smoothness, we present a simple and efficient method to compute the spatial and angular scaling functions (§6).

- To enable level-of-detail (LoD) rendering, we present a linear interpolation method that provides smooth transitions between our models prefiltered at varying scales (\$7.2).

\section{RELATED WORK}

We review several main research areas in the following paragraphs. Comparison between our method and the most related techniques is summarized in Table 1.

Surface appearance prefiltering. We refer readers to [Bruneton and Neyret 2012] for a comprehensive review of surface appearance prefiltering techniques. Traditional normal/BRDF map filtering methods [Fournier 1992; Tan et al. 2005; Toksvig 2005; Han et al. 


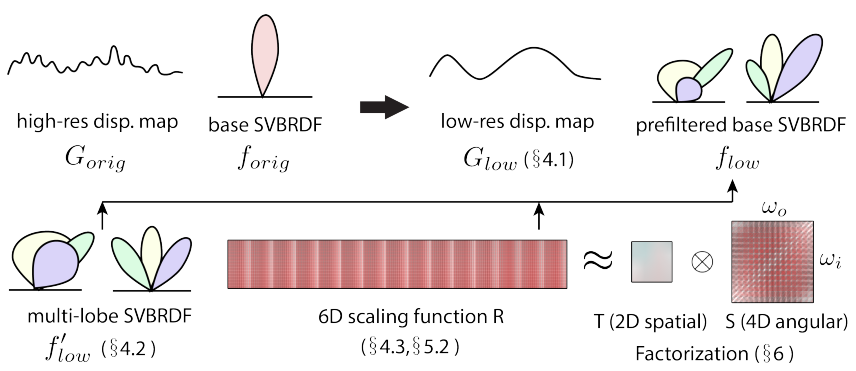

Fig. 3. Workflow of our method. We convert a high-resolution displacement map and base BRDF (top-left) to a low-resolution one and prefiltered SVBRDF (top-right). The prefiltered SVBRDF is obtained as a product of the SVBRDF from a multi-lobe NDF (bottom-left) and a 6D scaling function (bottom-right). This 6D function can be further factorized as the product of a single 2D spatial scaling function and a single $4 \mathrm{D}$ angular scaling function.

2007; Kaplanyan et al. 2016; Xu et al. 2017] ignore shadowing and masking effects. Tan et al. [2008] approximate shadowing and masking using horizon map distributions. LEADR [Dupuy et al. 2013] extends from LEAN mapping [Olano and Baker 2010] by incorporating a physically based shadowing-masking term derived from microfacet theory. In LEADR, they use one-lobe NDFs for simplicity and efficiency in a real-time rendering context. We use multi-lobe NDFs to achieve better accuracy, especially at glossy highlights (Figure 16). Recently, Loubet and Neyret [2017] proposed a hybrid mesh-volume model to construct LoDs of complex objects. They leverage volumetric models to handle self-occlusions caused by micro-geometries, while our method generates pure surface reflectance models that are simpler and more efficient to use. In addition, none of these methods take interreflections into account, since interreflections combined with shadowing and masking are complicated to analyze and prefilter.

Bidirectional texture functions (BTF) [Cabral et al. 1987; Dana et al. 1999] capture spatially varying and view-dependent surface appearance. Generating 6D BTF data requires expensive precomputation and a large amount of storage. Although BTF filtering methods [Ma et al. 2005; Wu et al. 2009; Jarabo et al. 2014] provide a direct solution to surface appearance prefiltering, the high dimensionality limits their practical applications. Our method separates a full $6 \mathrm{D}$ function into a $4 \mathrm{D}$ SVNDF and a $6 \mathrm{D}$ scaling function. The latter scaling function can be further factorized into a single $2 \mathrm{D}$ spatial scaling function and a single 4D angular scaling function, which is cheaper to compute and compact to store. Complex shadowingmasking and interreflections can be captured by the spatial and angular scaling functions accurately.

Bi-scale material design. A series of works model object's macroscale appearance by manipulating its micro-scale details [Westin et al. 1992; Heidrich et al. 2000; Wu et al. 2011; Iwasaki et al. 2012]. Westin et al. [1992] explicitly simulate ray tracing on micro-geometry. Heidrich et al. [2000] speed up the simulation on height fields using precomputed visibility. Wu et al. [2011] propose an interactive biscale material editing system. They precompute rotated BRDF values and bidirectional visible normal distribution functions (BVNDF) and compress them as low-rank matrices. This work has been extended
Table 1. We compare our method with LEADR [Dupuy et al. 2013], biscale appearance models [Wu et al. 2011; Iwasaki et al. 2012] and multiscattering microfacet models [Heitz et al. 2016; Lee et al. 2018; Xie and Hanrahan 2018]. Our method can handle both shadowing-masking and interreflections but is not limited to Gaussian/GGX surfaces, at the expense of some precomputation.

\begin{tabular}{|c|c|c|c|c|}
\hline Method & LEADR & bi-scale & microfacet & ours \\
\hline Shadowing and masking & $\checkmark$ & $\checkmark$ & $\checkmark$ & $\checkmark$ \\
\hline Interreflections & $x$ & $x$ & $\checkmark$ & $\checkmark$ \\
\hline General surfaces & $x$ & $\checkmark$ & $x$ & $\checkmark$ \\
\hline No Precomputation & $\checkmark$ & $x$ & $\checkmark$ & $x$ \\
\hline
\end{tabular}

to edit highly glossy materials by using mixtures of spherical Gaussians (SG) or anisotropic spherical Gaussians (ASG) [Iwasaki et al. 2012; Xu et al. 2013]. The shadowing-masking term is controlled by the weights of SG/ASG lobes. Most of these bi-scale appearance modeling techniques focus on the average large-scale appearance and do not consider spatial variation. In addition, the interactive approaches have no interreflection components. Recently, several methods for rendering glinty surfaces have been developed by simulating specular reflection on micro-surfaces [Yan et al. 2014; Jakob et al. 2014; Yan et al. 2016; Chermain et al. 2018]. Zirr and Kaplanyan [2016] propose a bi-scale microfacet model to render micro-details in realtime. Their methods focus on spatially varying NDFs, neglecting shadowing-masking and interreflections.

Microfacet models. Microfacet models describe the aggregate reflectance from a statistical representation of rough surfaces, i.e. the orientations of microfacets, resulting in a number of physically based BRDFs [Cook and Torrance 1982; Oren and Nayar 1994; Walter et al. 2007]. The Smith model [Smith 1967; Heitz 2014] gives an accurate approximation of the microfacet shadowing-masking function with the assumption of independence between heights and normals. In particular, the shadowing-masking function has analytic solutions if Gaussian or GGX surfaces are given. Ashikmin et al. [2000] derive a $4 \mathrm{D}$ BRDF from a 2D NDF, in which the shadowing-masking term is numerically computed from the NDF. LEADR [Dupuy et al. 2013] assumes Gaussian surfaces and leverages the Smith model to handle masking and shadowing effects. Recent works [Heitz et al. 2016; Lee et al. 2018; Xie and Hanrahan 2018] extend microfacet theory by modeling multiple scattering inside the micro-surface. Though microfacet models enable efficient computation of shadowing-masking and interreflections, they are limited by Gaussian surfaces, GGX surfaces or V-grooves. Our method can handle general surfaces without assumptions for specific micro-geometries.

Inverse rendering. Inverse rendering optimizes for scene parameters that produce the best match to target appearance. Previous methods [Hašan and Ramamoorthi 2013; Gkioulekas et al. 2013; Khungurn et al. 2015; Zhao et al. 2016; Gkioulekas et al. 2016] require global inverse rendering, which solves for a number of scattering parameters and involves expensive Monte Carlo path tracing during iterative optimizations. We just need to perform standard normal mapping and precompute simpler effective BRDFs once to obtain the prefiltered scattering parameters. Unlike inverse rendering techniques that depend on specific scene configurations, our model can 
Table 2. Definitions of commonly used symbols.

\begin{tabular}{|c|c|c|}
\hline Symbol & Meaning & Def. \\
\hline $\mathscr{P}$ & Surface patch & $\S 3$ \\
\hline $\mathcal{G}(\mathcal{P})$ & $\begin{array}{l}\text { Micro-geometry defined by a surface } \\
\text { patch (subset) } \mathcal{P} \text { of a displacement map }\end{array}$ & $\S 3$ \\
\hline$f$ & Isotropic base BRDF & $\S 3$ \\
\hline$f^{\mathrm{eff}}(\mathcal{G}, f)$ & $\begin{array}{l}\text { Effective BRDF determined by } \\
\text { micro-geometry } \mathcal{G} \text { and base BRDF } f\end{array}$ & $\S 3$, Eq. (6) \\
\hline $\mathcal{G}_{\text {orig }}$ & $\begin{array}{l}\text { Micro-geometry defined by the original } \\
\text { high-resolution displacement map }\end{array}$ & $\S 4$ \\
\hline$f_{\text {orig }}$ & Original input base BRDF & $\S 4$ \\
\hline $\mathcal{G}_{\text {low }}$ & $\begin{array}{l}\text { Micro-geometry defined by the } \\
\text { low-resolution displacement map }\end{array}$ & $\S 4$ \\
\hline$f_{\text {low }}$ & Prefiltered spatially varying base BRDF & $\S 4$ \\
\hline$f_{\text {low }}^{\prime}$ & Spatially varying multi-lobe BRDF & $\S 4.2$, Eq. (11) \\
\hline$f_{\text {ir }}^{\text {eff }}$ & Effective BRDF with interreflections & §5.1, Eq. (17) \\
\hline$R, R_{\mathrm{ir}}$ & $\begin{array}{l}\text { 6D scaling function } \\
\text { without/with interreflections }\end{array}$ & $\begin{array}{l}\text { §4.3, Eq. (12) } \\
\text { §5.2, Eq. (20) }\end{array}$ \\
\hline$T, T_{\mathrm{ir}}$ & $\begin{array}{l}\text { 2D spatial scaling function } \\
\text { without/with interreflections }\end{array}$ & $\begin{array}{l}\text { §4.3, Eq. (14) } \\
\text { §6, Eq. (21) }\end{array}$ \\
\hline$S, S_{\text {ir }}$ & $\begin{array}{l}\text { 4D angular scaling function } \\
\text { without/with interreflections }\end{array}$ & $\begin{array}{l}\text { §4.3, Eq. (14) } \\
\text { §6, Eq. (21) }\end{array}$ \\
\hline
\end{tabular}

generalize to different lighting and viewing conditions as we match the effective BRDFs rather than rendered images.

Height field rendering. Rendering displacement-mapped surfaces closely relates to height field rendering. Self-shadowing on height fields can be computed in real-time by determining horizon angles for a set of azimuthal directions [Snyder and Nowrouzezahrai 2008; Timonen and Westerholm 2010]. Fast height field rendering with global illumination [Nowrouzezahrai and Snyder 2009] approximates visibility and indirect radiance using low-order $\mathrm{SH}$ basis functions, which cannot capture high-frequency reflection and shadowing effects. In these methods, height fields are attached on a planar base surface. Our method allows perturbing displacements on a general surface representation such as triangular meshes.

\section{PRELIMINARIES}

In this section, we provide preliminaries of displacement mapping, normal mapping, and effective BRDFs. Table 2 summarizes all symbols commonly used in this paper.

Displacement mapping. Displacement maps provide an efficient way to describe detailed micro-geometries. Mathematically, a displacement map is a function $h:[0,1]^{2} \rightarrow \mathbb{R}$ that specifies the distance which individual surface points are shifted along the normal directions. To be precise, given a texture-mapped base surface and a displacement map, the resulting geometry is obtained by moving each surface point with texture coordinate $(u, v)$ along its normal direction for a distance of $h(u, v)$. Further, a surface patch $\mathcal{P} \subseteq[0,1]^{2}$ denotes a small and locally flat region of the base surface. For notational simplicity, the patch area is normalized using some filter kernel $k_{\mathcal{P}}{ }^{1}$ (i.e., $\int_{\mathcal{P}} k_{\mathcal{P}}(\boldsymbol{p}) \mathrm{d} \boldsymbol{p}=1$ ). For each $\boldsymbol{p} \in \mathcal{P}$, we

\footnotetext{
${ }^{1}$ We use a box filter in this paper.
}

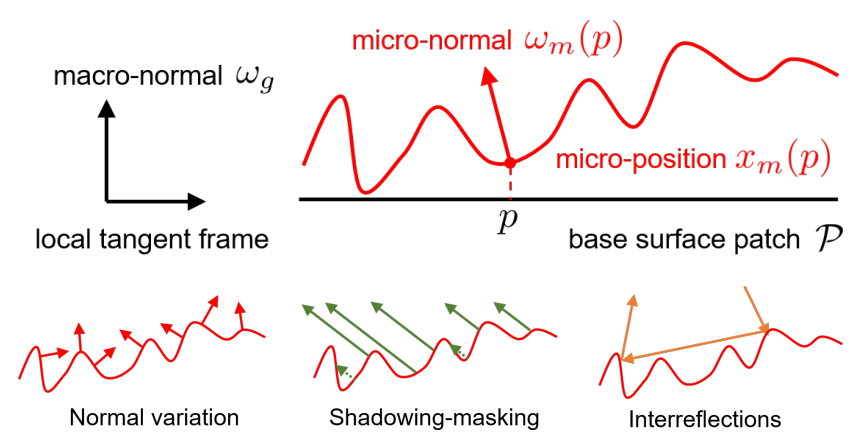

Fig. 4. Illustrations of micro-geometry and related illumination effects. They are shown in 2D for simplicity, but fully developed in 3D.

denote its final position (perturbed by displacement mapping) and micro-normal as $\boldsymbol{x}_{m}(\boldsymbol{p})$ and $\boldsymbol{\omega}_{m}(\boldsymbol{p})$, respectively. Lastly, the microgeometry given by a base patch $\mathcal{P}$ and a displacement map can be expressed by a collection of micro-positions and micro-normals: $\mathcal{G}(\mathcal{P})=\left\{\left(\boldsymbol{x}_{m}(\boldsymbol{p}), \omega_{m}(\boldsymbol{p})\right) \mid \boldsymbol{p} \in \mathcal{P}\right\}$.

Meso-geometry. For a surface patch $\mathcal{P}$, we define a local tangent frame under which the macro-normal $\omega_{g}$ of the patch equals to $(0,0,1)$. Then, the meso-scale geometry of $\mathcal{G}(\mathcal{P})$ can be described with the average slope:

$$
\tilde{\boldsymbol{s}}=\int_{\mathcal{P}} \boldsymbol{s}_{m}(\boldsymbol{p}) k_{\mathcal{P}}(\boldsymbol{p}) \mathrm{d} \boldsymbol{p},
$$

where $\boldsymbol{s}_{m}(\boldsymbol{p})=\left(-x_{m} / z_{m},-y_{m} / z_{m}\right)$ is the micro-slope with $x_{m}, y_{m}$, and $z_{m}$ given by the micro-normal at $\boldsymbol{p}$ (i.e., $\left.\left(x_{m}, y_{m}, z_{m}\right)=\omega_{m}(\boldsymbol{p})\right)$ under the local tangent frame.

Normal mapping. Provided a displacement map, we can extract the normal distribution function (NDF) [Dupuy et al. 2013] for any given surface patch $\mathcal{P}$ via

$$
D(\omega)=\int_{\mathcal{P}} \frac{\delta\left(\omega-\omega_{m}(\boldsymbol{p})\right)}{\left\langle\omega_{m}(\boldsymbol{p}), \omega_{g}\right\rangle} k_{\mathcal{P}}(\boldsymbol{p}) \mathrm{d} \boldsymbol{p}
$$

where $\langle\cdot, \cdot\rangle$ represents the dot product clamped to 0 , and $\delta$ denotes the Dirac delta function. A physically valid NDF must satisfy

$$
\int_{\mathcal{H}^{2}} D(\omega)\left\langle\omega, \omega_{g}\right\rangle \mathrm{d} \omega=\int_{\mathcal{P}} k_{\mathcal{P}}(\boldsymbol{p}) \mathrm{d} \boldsymbol{p}=1,
$$

where $\mathcal{H}^{2}$ denotes the unit hemispherical domain. It means that the projected area of the micro-geometry onto the macro-normal $\omega_{g}$ should be equal to the area of the base surface patch.

An NDF $D$ can be approximated by a mixture of von Mises-Fisher (vMF) lobes [Han et al. 2007],

$$
D(\boldsymbol{\omega}) \approx \sum_{i=1}^{m} \alpha_{i} \gamma\left(\boldsymbol{\omega} ; \kappa_{i}, \boldsymbol{\mu}_{i}\right),
$$

where $\alpha_{i}$ represents the lobe's amplitude. Each vMF lobe with bandwidth $\kappa_{i}$ and the center direction $\mu_{i}$ is defined as $\gamma\left(\omega ; \kappa_{i}, \mu_{i}\right)=$ $\frac{\kappa_{i}}{4 \pi \sinh \kappa_{i}} \exp \left(\kappa_{i}\left(\boldsymbol{\omega} \cdot \boldsymbol{\mu}_{i}\right)\right)$. This vMF-based parameterization allows efficient description of spatially varying NDFs (SVNDFs).

From an NDF $D$ and an isotropic base $\operatorname{BRDF} f$, the composite multi-lobe BRDF can be formulated as [Han et al. 2007]

$\rho\left(\omega_{i}, \omega_{o} ; D, f\right)=\frac{1}{\cos \theta_{i}} \int_{\mathcal{H}^{2}} f\left(R_{\omega}\left(\omega_{i}\right), R_{\omega}\left(\omega_{o}\right)\right)\left\langle\omega, \omega_{i}\right\rangle D(\omega) \mathrm{d} \omega$. (5) 
Note that $\omega_{i}$ and $\omega_{o}$ are in the macro-scale tangent frame defined by the macro-normal $\omega_{g}$. We need a rotation function $R_{\omega}(\cdot)$ to transform $\omega_{i}$ and $\omega_{o}$ to the local frame defined by the micro-normal $\omega$. The multi-lobe BRDF keeps the orientations of the micro-normals, but neglects both shadowing-masking and interreflections caused by the micro-geometry.

Effective BRDFs. To include shadowing-masking effects, the effective BRDF $f^{\text {eff }}$ is used to describe the overall reflectance of a patch $\mathcal{P}$ [Wu et al. 2011]. It depends on the micro-geometry $\mathcal{G}(\mathcal{P})$ and the micro-BRDF $f$. The effective BRDF can be viewed as the average of the cosine-weighted and shadowed micro-BRDFs weighted by the visible projected area along the viewing direction $\omega_{o}$ :

$$
\begin{array}{r}
f^{\mathrm{eff}}\left(\boldsymbol{\omega}_{i}, \omega_{o} ; \mathcal{G}, f\right)=\frac{1}{A_{\mathcal{G}}\left(\omega_{o}\right)} \int_{\mathcal{P}} f\left(\boldsymbol{x}_{m}(\boldsymbol{p}), \boldsymbol{\omega}_{i}, \omega_{o}\right)\left\langle\boldsymbol{\omega}_{m}(\boldsymbol{p}), \boldsymbol{\omega}_{i}\right\rangle \\
V\left(\boldsymbol{x}_{m}(\boldsymbol{p}), \omega_{i}\right) A_{\mathcal{G}}\left(\boldsymbol{p}, \omega_{o}\right) k_{\mathcal{P}}(\boldsymbol{p}) \mathrm{d} \boldsymbol{p},
\end{array}
$$

where $V(x, \omega)$ denotes the binary visibility function (indicating whether a ray starting from point $\boldsymbol{x}$ along direction $\omega$ is occluded), and $A_{\mathcal{G}}\left(p, \omega_{o}\right)$ is the visible projected area along $\omega_{o}$ given by

$$
A_{\mathcal{G}}\left(\boldsymbol{p}, \omega_{o}\right)=\frac{\left\langle\omega_{o}, \omega_{m}(p)\right\rangle}{\left\langle\omega_{g}, \omega_{m}(p)\right\rangle} V\left(\boldsymbol{x}_{m}(\boldsymbol{p}), \omega_{o}\right)
$$

The total visible projected area of $\mathcal{G}(\mathcal{P})$ is the normalization factor of the weighted average [Dupuy et al. 2013; Heitz 2014],

$A_{\mathcal{G}}\left(\omega_{o}\right)=\int_{\mathcal{P}} \frac{\left\langle\omega_{o}, \omega_{m}(p)\right\rangle}{\left\langle\omega_{g}, \omega_{m}(p)\right\rangle} V\left(x_{m}(p), \omega_{o}\right) k_{\mathcal{P}}(\boldsymbol{p}) \mathrm{d} \boldsymbol{p}=\frac{\left\langle\omega_{o}, \omega_{n}\right\rangle}{\left\langle\omega_{g}, \omega_{n}\right\rangle}$,

where $\omega_{n}$ denotes the meso-scale normal direction.

The effective BRDF (6) has the cosine term and the visibilities baked in and captures the shadowing-masking caused by microscale self-occlusions. We will generalize the formulation in $\S 5.1$ to capture interreflections.

\section{PREFILTERING REFLECTANCE PARAMETERS}

Our method takes as input a high-resolution displacement map $h_{\text {orig }}$ and an isotropic base micro-BRDF $f_{\text {orig }}$ (which could be spatially varying, e.g., Figure 14). We denote $\mathcal{G}_{\text {orig }}(\mathcal{P})$ as the micro-geometry defined by the original displacement map on a surface patch $\mathcal{P}$. Then, we prefilter the input model and obtain a lower-resolution displacement map $h_{\text {low }}$ (with $\mathcal{G}_{\text {low }}(\mathcal{P})$ representing its micro-geometry on $\mathcal{P}$ ) associated with a new spatially varying BRDF $f_{\text {low }}$. Our goal is to have the appearance of the prefiltered model closely resemble the input. Notice that, even if the input base BRDF $f_{\text {orig }}$ is spatially invariant, the prefiltered reflectance $f_{\text {low }}$ may need to have spatial variations to accurately reproduce the detailed appearance of the input model.

To this end, our technique starts with computing the downsampled displacement map $h_{\text {low }}$ by minimizing differences of the mesoscale slopes (§4.1). Then, we seek a prefiltered SVBRDF $f_{\text {low }}$ that preserves the original appearance for the downsampled displacement map. This, however, is nontrivial as $f_{\text {low }}$ generally needs to be spatially varying. We introduce a novel two-step approach to compute $f_{\text {low }}$. First, for each base patch of the downsampled displacement map $h_{\text {low }}$, we compute its corresponding patch NDF from the original displacement map $h_{\text {orig. }}$ Each patch NDF implies a spatially varying multi-lobe BRDF without considering shadowingmasking and interreflections. This step generates a spatially varying base BRDF $f_{\text {low }}^{\prime}$ as an initial solution (§4.2). Then, we scale $f_{\text {low }}^{\prime}$ by a 6D scaling function $R\left(\boldsymbol{x}, \omega_{i}, \omega_{o}\right)$ to match the effective BRDFs ( $\left.§ 4.3\right)$. The final prefiltered SVBRDF $f_{\text {low }}=R \cdot f_{\text {low }}^{\prime}$ is able to reproduce the original appearance. Our method is also illustrated in Figure 3.

Although the scaling function $R\left(\boldsymbol{x}, \omega_{i}, \omega_{o}\right)$ is $6 \mathrm{D}$, we will show in $\S 6$ that $R$ can be factorized into a 2D spatial function of location $\boldsymbol{x}$ and a 4D angular function of directions $\omega_{i}$ and $\omega_{o}$. Further, these functions are usually smooth in practice, and therefore can be computed efficiently as low-resolution tabulated functions, allowing our prefiltered models to be compactly represented.

\subsection{Downsampling Displacement Maps}

As stated in $\S 3$, displacement maps are defined mathematically as continuous 2D scalar functions. In practice, we represent displacement maps as piecewise linear functions using 2D textures. Specifically, the original high-resolution displacement map $h_{\text {orig }}$ is defined at each vertex of a dense grid: $\left\{\left(u \cdot 2^{-l}, v \cdot 2^{-l}\right)\right\}$ for $u, v=0, \ldots, 2^{l}$. The prefiltered low-resolution displacement map $h_{\text {low }}$ uses a coarse grid $\left\{\left(u \cdot 2^{-l^{\prime}}, v \cdot 2^{-l^{\prime}}\right)\right\}$ for $u, v=0, \ldots, 2^{l^{\prime}}$ with some $l^{\prime}<l$.

Obtaining $h_{\text {low }}$ requires specifying the displacement values at the vertices of the coarse grid. For every patch $\mathcal{P}_{u v}=\left[u \cdot 2^{-l^{\prime}},(u+1)\right.$. $\left.2^{-l^{\prime}}\right] \times\left[v \cdot 2^{-l^{\prime}},(v+1) \cdot 2^{-l^{\prime}}\right]$ covering a grid cell of $h_{\text {low }}$, our goal is to find out the optimal heights at its four corners (i.e., grid points) such that the average slope $\tilde{\boldsymbol{s}}_{u v}$ of the bilinear patch closely matches the reference slope $\tilde{\boldsymbol{s}}_{u v}^{*}$ of the original micro-surface $\mathcal{G}_{\text {orig }}\left(\mathcal{P}_{u v}\right)$. The reference average slope $\tilde{\boldsymbol{s}}_{u v}^{*}$ can be calculated using Eq. (1). On the other hand, the average slope of the bilinear patch $\mathcal{P}_{u v}$ is

$$
\tilde{\boldsymbol{s}}_{u v}=\left(\frac{h_{11}+h_{10}-h_{01}-h_{00}}{2}, \frac{h_{11}+h_{01}-h_{10}-h_{00}}{2}\right),
$$

where $h_{00}, h_{10}, h_{01}, h_{11}$ denote the displacement values at the four cell corners. Please refer to Appendix A for the derivation. To minimize the differences between the two average slopes robustly, we further introduce a regularization term for local smoothness, yielding the final objective function:

$$
L\left(h_{\mathrm{low}}\right)=\sum_{u} \sum_{v}\left\|\tilde{\boldsymbol{s}}_{u v}-\tilde{\boldsymbol{s}}_{u v}^{*}\right\|^{2}+w\left\|\Delta h_{\mathrm{low}}\left(\frac{u}{2^{l^{\prime}}}, \frac{v}{2^{l^{\prime}}}\right)\right\|^{2},
$$

where $\Delta$ is the Laplacian operator and $w=0.01$ is the weight of the regularization term. By minimizing this objective function using least-squares, the optimal solution gives the downsampled displacement map $h_{\text {low }}$ and its corresponding micro-geometry $\mathcal{G}_{\text {low }}$.

\subsection{Spatially Varying Multi-Lobe BRDF}

For a cell from the coarse grid of the downsampled displacement map $h_{\text {low }}$ and its corresponding patch $\mathcal{P}_{u v}$, the patch NDF $D_{u v}(\omega)$ defined in Eq. (2) provides a compact approximation of the original micro-geometry $\mathcal{G}_{\text {orig }}\left(\mathcal{P}_{u v}\right)$. Previous works such as LEADR [Dupuy et al. 2013] typically assume the NDFs to follow Beckmann distributions. Our method, in contrast, does not enforce any restriction on the NDFs and is therefore more general. We use the normal mapping technique [Han et al. 2007] to fit an NDF with a mixture of vMF lobes (4), resulting in a spatially varying NDF at a lower resolution. The SVNDF is technically 4D but can be described compactly using spatially varying vMF parameters (stored as $2 \mathrm{D}$ textures). 
From these NDFs that approximate $\mathcal{G}_{\text {orig }}$ and the original base micro-BRDF $f_{\text {orig, }}$, we use Eq. (5) to formulate the initial multi-lobe BRDF for each patch $\mathcal{P}_{u v}$ :

$$
f_{\text {low }}^{\prime}\left(x, \omega_{i}, \omega_{o}\right)=\rho\left(\omega_{i}, \omega_{o} ; D_{u v}, f_{\text {orig }}\right) .
$$

Here the micro-position $x$ is within the patch $\mathcal{P}_{u v}$.

\subsection{Scaling Function}

Although the multi-lobe SVBRDF $f_{\text {low }}^{\prime}$ is a good start for matching the input appearance, it is incomplete as it neglects the shadowingmasking and interreflection effects. To address this problem, we first consider the single-bounce case (i.e., direct illumination that only involves shadowing and masking). We will discuss the multiplebounce case that handles interreflections in §5.

To capture shadowing and masking, we multiply the initial multilobe SVBRDF $f_{\text {low }}^{\prime}$ with another scaling function $R$. Suppose $R$ has a spatial resolution of $M^{2}$, we uniformly subdivide the base surface into $M^{2}$ patches. Let $\mathcal{P}_{\boldsymbol{x}}$ denote the patch containing $\boldsymbol{x}$. We define the scaling function $R\left(x, \omega_{i}, \omega_{o}\right)$ as the ratio ${ }^{2}$ between the original effective BRDF (6) and the prefiltered effective BRDF over $\mathcal{P}_{\boldsymbol{x}}$ :

$$
R\left(\boldsymbol{x}, \omega_{i}, \omega_{o}\right)=\frac{f^{\text {eff }}\left(\omega_{i}, \omega_{o} ; \mathcal{G}_{\text {orig }}\left(\mathcal{P}_{\boldsymbol{x}}\right), f_{\text {orig }}\right)}{f^{\text {eff }}\left(\omega_{i}, \omega_{o} ; \mathcal{G}_{\text {low }}\left(\mathcal{P}_{\boldsymbol{x}}\right), f_{\text {low }}^{\prime}\right)},
$$

The final prefiltered base SVBRDF can then be expressed as

$$
f_{\text {low }}\left(x, \omega_{i}, \omega_{o}\right)=R\left(x, \omega_{i}, \omega_{o}\right) f_{\text {low }}^{\prime}\left(x, \omega_{i}, \omega_{o}\right) .
$$

It is easy to verify that the final effective BRDF $f^{\text {eff }}\left(\mathcal{G}_{\text {low }}, f_{\text {low }}\right)$ (with $\omega_{i}$ and $\omega_{o}$ omitted for notational simplicity) over $\mathcal{P}_{\boldsymbol{x}}$ equals the original effective BRDF, since the single-bounce effective BRDF is linear in the scaling factors, i.e., $f^{\text {eff }}\left(\mathcal{G}_{\text {low }}, R \cdot f_{\text {low }}^{\prime}\right)=R \cdot f^{\text {eff }}\left(\mathcal{G}_{\text {low }}, f_{\text {low }}^{\prime}\right)$. The identical effective BRDFs indicate a good match of aggregate reflectance between the original and the prefiltered models.

Due to the high dimensionality of the scaling function $R$, it is challenging to obtain and store. We will show in $\S 6$ that $R$ can be accurately approximated as the product of a function $T(\boldsymbol{x})$ of location and a function $S\left(\omega_{i}, \omega_{o}\right)$ of directions:

$$
R\left(\boldsymbol{x}, \boldsymbol{\omega}_{i}, \boldsymbol{\omega}_{o}\right) \approx T(\boldsymbol{x}) \cdot S\left(\boldsymbol{\omega}_{i}, \boldsymbol{\omega}_{o}\right) .
$$

We first generalize our scaling function to interreflections in $\S 5$ Then, in $\S 6$, we develop the factorization above and show that the factorized scaling functions $T$ and $S$ can be computed directly without fully computing the 6D function $R$.

\section{INTERREFLECTIONS}

We now describe how to capture interreflection effects using a scaling function similar to Eq. (12). We first generalize the effective BRDF formulation (6) to measure the average surface reflectance with interreflections (§5.1). Then, we provide an approximate solution to the scaling function with interreflections handled (§5.2).

\footnotetext{
${ }^{2}$ In practice, we need to add a small $\epsilon$ to avoid division by zero.
}

\subsection{Effective BRDF with Interreflections}

The effective BRDF formulation depicted in $\$ 3$ models only direct (i.e., single-bounce) illumination of the micro-geometry. This leads to significant energy loss due to the neglect of interreflections within the micro-geometry. To address this problem, we adapt Veach's path integral formulation [1997] to express effective BRDFs with interreflections.

A ray that bounces within the micro-geometry $\mathcal{G}=\left\{\left(\boldsymbol{x}_{m}, \omega_{m}\right)\right\}$ can be described by a light transport path $\overline{\boldsymbol{x}}=\left(\boldsymbol{x}_{1}, \boldsymbol{x}_{2}, \ldots, \boldsymbol{x}_{k}\right)$ as well as the viewing direction $\omega_{o}$ and the lighting direction $\omega_{i}$. Notice that, unlike the traditional formulation where the endpoints of a path respectively lay on the light source and the sensor, all the path vertices $x_{1}, \ldots, x_{k}$ are located on the micro-surface in our case. The path contribution $h(\overline{\boldsymbol{x}})$ is given by the product of BRDF terms and cosine terms, followed by a visibility term at the last path vertex $\boldsymbol{x}_{k}$ along the lighting direction $\boldsymbol{\omega}_{i}$ :

$h(\overline{\boldsymbol{x}})=V\left(\boldsymbol{x}_{k}, \boldsymbol{\omega}_{i}\right) \prod_{j=1}^{k} f\left(\boldsymbol{x}_{j}, \boldsymbol{\omega}_{j \rightarrow j+1}, \boldsymbol{\omega}_{j \rightarrow j-1}\right)\left\langle\boldsymbol{n}\left(\boldsymbol{x}_{j}\right), \boldsymbol{\omega}_{j \rightarrow j+1}\right\rangle$,

where $\omega_{i \rightarrow j}:=\frac{\boldsymbol{x}_{j}-\boldsymbol{x}_{\boldsymbol{i}}}{\left\|\boldsymbol{x}_{j}-\boldsymbol{x}_{i}\right\|}$ indicates the normalized direction from $\boldsymbol{x}_{i}$ to $x_{j}$ (with $\omega_{1 \rightarrow 0}:=\omega_{o}$ and $\omega_{k \rightarrow k+1}:=\omega_{i}$ ), and $\boldsymbol{n}\left(x_{j}\right)$ denotes the micro-normal at $\boldsymbol{x}_{\boldsymbol{j}}$.

We define the path space $\Omega\left(x, \omega_{i}, \omega_{o}\right)$ as a collection of light transport paths $\overline{\boldsymbol{x}}=\left(\boldsymbol{x}_{1}, \ldots, \boldsymbol{x}_{k}\right)$ with $\boldsymbol{x}_{1}=\boldsymbol{x}$, and the viewing and illumination directions given by $\omega_{o}$ and $\omega_{i}$, respectively. At a given position $\boldsymbol{x}_{m}(\boldsymbol{p}) \in \mathcal{G}$, the reflectance including interreflections aggregates contributions from all the valid paths:

$$
r\left(\boldsymbol{x}_{m}(\boldsymbol{p}), \boldsymbol{\omega}_{i}, \boldsymbol{\omega}_{o}\right)=\int_{\Omega\left(\boldsymbol{x}_{m}(\boldsymbol{p}), \omega_{i}, \omega_{o}\right)} h(\overline{\boldsymbol{x}}) \mathrm{d} \mu(\overline{\boldsymbol{x}}),
$$

where $\mathrm{d} \mu(\overline{\boldsymbol{x}})$ denotes the path throughput measure, which is a product of solid angle measures on the path directions.

According to Eqs. (6) and (16), we express the multi-bounce effective BRDF as the average path contribution over $\mathcal{G}$ weighted by the visible projected area along $\omega_{0}$ :

$f_{\text {ir }}^{\text {eff }}\left(\omega_{i}, \omega_{o} ; \mathcal{G}, f\right)=\frac{1}{A_{\mathcal{G}}\left(\omega_{o}\right)} \int_{\mathcal{P}} r\left(\boldsymbol{x}_{m}(\boldsymbol{p}), \omega_{i}, \omega_{o}\right) A_{\mathcal{G}}\left(\boldsymbol{p}, \omega_{o}\right) k_{\mathcal{P}}(\boldsymbol{p}) \mathrm{d} \boldsymbol{p}$.

Under this formulation, the single-bounce effective BRDF (6) becomes a special case where each path $\overline{\boldsymbol{x}}$ is restricted to contain only one vertex.

\subsection{Computing the Scaling Function}

Following a similar idea as $\S 4.3$, we scale the initial multi-lobe SVBRDF $f_{\text {low }}^{\prime}$ by a scaling function $R_{\text {ir }}$ :

$$
f_{\text {low }}\left(x, \omega_{i}, \omega_{o}\right)=R_{\mathrm{ir}}\left(x, \omega_{i}, \omega_{o}\right) f_{\text {low }}^{\prime}\left(x, \omega_{i}, \omega_{o}\right),
$$

so that the resulting multi-bounce effective BRDF (17) of the prefiltered model matches that of the input. Namely, $f_{\text {ir }}^{\text {eff }}\left(\mathcal{G}_{\text {low }}, f_{\text {low }}\right)=$ $f_{\text {ir }}^{\text {eff }}\left(\mathcal{G}_{\text {orig }}, f_{\text {orig }}\right)$. Since $f_{\text {ir }}^{\text {eff }}$ is nonlinear in $R_{\text {ir }}$ due to multiple scattering (i.e., $f_{\text {ir }}^{\text {eff }}\left(\mathcal{G}_{\text {low }}, R_{\text {ir }} \cdot f_{\text {low }}^{\prime}\right) \neq R_{\text {ir }} \cdot f_{\text {ir }}^{\text {eff }}\left(\mathcal{G}_{\text {low }}, f_{\text {low }}^{\prime}\right)$ in general), $R_{\mathrm{ir}}$ cannot be obtained using Eq. (12). Although one can optimize $R_{\mathrm{ir}}$ iteratively using inverse rendering methods (e.g., [Khungurn et al. 2015; Zhao et al. 2016]), the optimization would be very expensive, if not impractical, due to the large number of unknowns involved. 


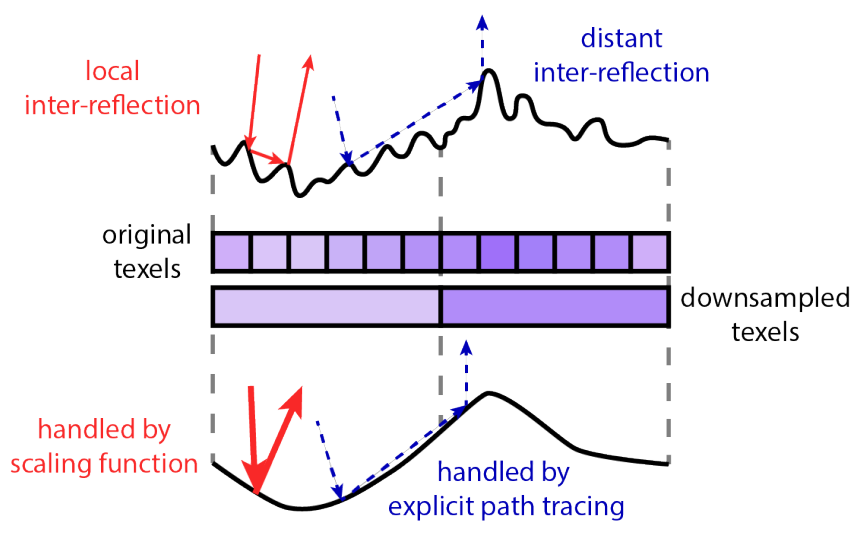

Fig. 5. Local and global interreflections. Our method tries to match local interreflections using a scaling function, while the path tracer handles distant (global) interreflections.

Instead, we seek $R_{\text {ir }}$ without performing global optimizations. To this end, we make a simplifying assumption: the high-order components in $f_{\text {ir }}^{\text {eff }}\left(\mathcal{G}_{\text {low }}, f_{\text {low }}\right)$ are negligible. In other words,

$$
f_{\text {ir }}^{\text {eff }}\left(\mathcal{G}_{\text {low }}, f_{\text {low }}\right) \approx f^{\text {eff }}\left(\mathcal{G}_{\text {low }}, f_{\text {low }}\right) .
$$

This is because the high-order component mainly consists of distant interreflections (i.e., those across multiple surface patches at the reduced resolution) that are supposed to be handled with explicit path tracing (see the blue arrows in Figure 5). The local interreflection, on the other hand, is usually weakened due to the downsampling of an object's micro-geometry, which we compensate using the scaling function $R_{\text {ir }}$ (see the red arrows in Figure 5).

Under this formulation, our goal becomes to match $f_{\text {ir }}^{\text {eff }}\left(\mathcal{G}_{\text {orig }}, f_{\text {orig }}\right)$ and $R_{\mathrm{ir}} \cdot f^{\mathrm{eff}}\left(\mathcal{G}_{\text {low }}, f_{\text {low }}^{\prime}\right)$, yielding the scaling function:

$$
R_{\mathrm{ir}}\left(\boldsymbol{x}, \omega_{i}, \omega_{o}\right)=\frac{f_{\mathrm{ir}}^{\mathrm{eff}}\left(\omega_{i}, \omega_{o} ; \mathcal{G}_{\mathrm{orig}}\left(\mathcal{P}_{\boldsymbol{x}}\right), f_{\mathrm{orig}}\right)}{f^{\mathrm{eff}}\left(\omega_{i}, \omega_{o} ; \mathcal{G}_{\mathrm{low}}\left(\mathcal{P}_{\boldsymbol{x}}\right), f_{\text {low }}^{\prime}\right)}
$$

In §8, we show that the simplification usually has minimal affect on accuracy (Figures 17, 18).

\section{PROPERTIES OF THE SCALING FUNCTION}

Upon establishing the scaling function $R_{\text {ir }}(20)$, our problem of prefiltering displacement-mapped surfaces boils down to efficiently representing and computing this function, ${ }^{3}$ which, unfortunately, is nontrivial as $R_{\mathrm{ir}}$ is $6 \mathrm{D}$ and generally too expensive to compute and store in brute-force ways.

We tackle this challenge by factorizing the $6 \mathrm{D}$ scaling function $R_{\mathrm{ir}}\left(\boldsymbol{x}, \omega_{i}, \omega_{o}\right)$ into a single spatial scaling function $T_{\mathrm{ir}}(\boldsymbol{x})$ and a single angular scaling function $S_{\mathrm{ir}}\left(\omega_{i}, \omega_{o}\right)$. In practice, both $T_{\mathrm{ir}}$ and $S_{\mathrm{ir}}$ are smooth (i.e., low-frequency). We exploit their smoothness to achieve efficient factorization using only a sparse sampling of $R_{\mathrm{ir}}$ (without explicitly precomputing the full $6 \mathrm{D}$ function). In the rest of this section, we provide more details on our factorization method.

${ }^{3}$ We use the notation $R_{\mathrm{ir}}$ in the multiple-bounce case since it is more general. The properties also hold for $R$ in the single-bounce case.

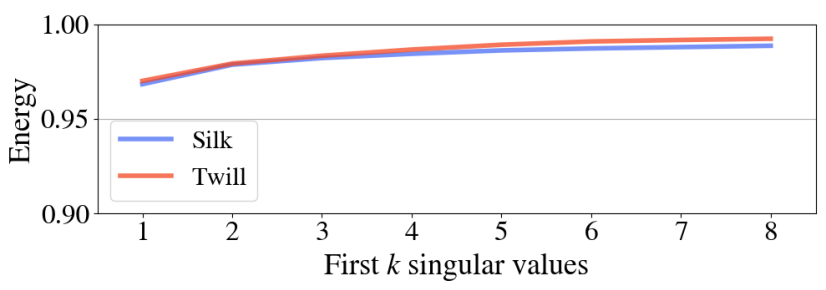

Fig. 6. Graph showing the fractions of energy carried by the top eight singular values of the scaling matrix.

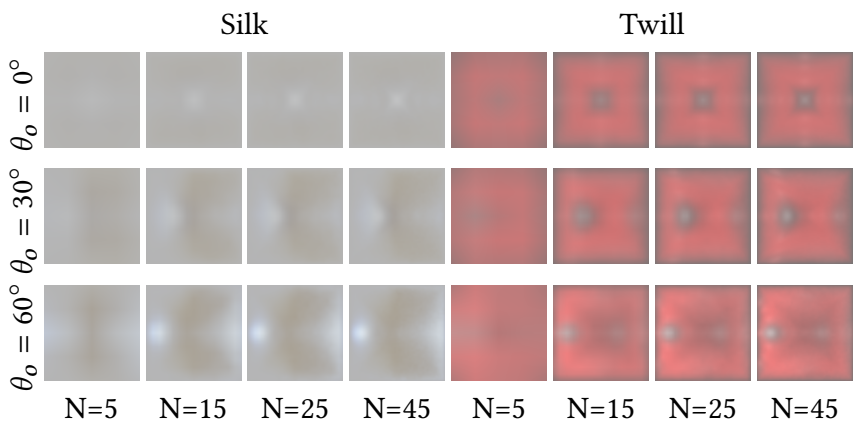

Fig. 7. Scaling function slices (with only $\omega_{i}$ changing and the other parameters fixed) for different angular resolutions. Using $N=15$ provides adequate accuracy for describing the scaling functions.

Rank-1 factorization. Our experiments indicate that the scaling functions are typically low-rank (Figure 6). To demonstrate this property, we compute the full 6D scaling functions for two example models: "silk" with only direct illumination; and "twill" with global illumination (details for these models are described in §8.2). In both cases, the scaling matrices $R_{\mathrm{ir}}$ are tabulated with a resolution $16^{2} \times$ $15^{2} \times 15^{2}\left(16^{2}\right.$ for $x, 15^{2}$ for $\omega_{i}, 15^{2}$ for $\left.\omega_{o}\right) .{ }^{4}$ After excluding the directions near grazing angles $\left(\theta>75^{\circ}\right),{ }^{5}$ we reshape $R_{\mathrm{ir}}$ into a $256 \times 11^{4}$ matrix, which is then decomposed using singular value decomposition (SVD). For both examples, the greatest singular value carries over $95 \%$ of the total energy (see Figure 6), suggesting that the scaling function $R_{\text {ir }}$ can indeed be accurately approximated by a rank-1 factorization:

$$
R_{\mathrm{ir}}\left(\boldsymbol{x}, \boldsymbol{\omega}_{i}, \boldsymbol{\omega}_{\mathrm{o}}\right) \approx T_{\mathrm{ir}}(\boldsymbol{x}) \cdot S_{\mathrm{ir}}\left(\boldsymbol{\omega}_{i}, \boldsymbol{\omega}_{o}\right) .
$$

Angular smoothness. We now seek the optimal resolution for the scaling function $R_{\mathrm{ir}}\left(\boldsymbol{x}, \omega_{i}, \omega_{o}\right)$ that provides a good balance between accuracy and data size, starting with the angular resolution $N$. Specifically, we use the concentric mapping [Shirley and Chiu 1997] to warp a unit hemisphere onto a unit square. Then, both $\omega_{i}$ are $\omega_{o}$ are sampled from a regular $2 \mathrm{D}$ grid with resolution $N^{2}$.

Because an effective BRDF captures the aggregate reflectance over a micro-surface, it generally has low angular frequency. Thus, the scaling function, as the ratio of two effective BRDFs, is also expected to be low-frequency angularly. Figure 7 shows $2 \mathrm{D}$ slices of the scaling functions (with $\omega_{i}$ changing and the other parameters fixed) for different angular resolutions $N=5,15,25$ and 45 (as references). The results indicate that $N=15$ provides good accuracy,

\footnotetext{
${ }^{4}$ This resolution is adequate due to the smoothness of $R_{\mathrm{ir}}$, which will be discussed later in this section.

${ }^{5}$ Numerical evaluation of the scaling functions is unstable near grazing angles.
} 
which is further supported by renderings in Figure 8. Therefore, we choose the resolution of $N=15$ for all main results in this paper.

Spatial smoothness. To determine the spatial resolution $M$, we perform a similar experiment by comparing renderings using resolutions of $M=1,4,16$. As shown in Figure 9, the results indicate that increasing the spatial resolution has diminishing benefits. This is because the scaling function varies in a very smooth fashion spatially. We thus use $M=4$ in most of our results (unless we state explicitly). In certain cases, even using $M=1$ (that causes the scaling function $T_{\mathrm{ir}}$ to be constant-valued) can offer sufficient accuracy.

Efficient factorization. Although computing the full 6D scaling function $R_{\mathrm{ir}}$ and decomposing it using SVD provides optimal factorization results, doing so is very costly since both steps require massive computation. We introduce a simple and efficient factorization method that constructs $T_{\mathrm{ir}}(\boldsymbol{x})$ and $S_{\mathrm{ir}}\left(\boldsymbol{\omega}_{i}, \boldsymbol{\omega}_{\mathrm{o}}\right)$ from sparse samples of $R_{\mathrm{ir}}$.

According to Eq. (21), $T_{\mathrm{ir}}(\boldsymbol{x})$ can be computed by averaging the $6 \mathrm{D}$ scaling function $R_{\mathrm{ir}}$ over the angular domain:

$$
T_{\mathrm{ir}}(\boldsymbol{x})=\frac{1}{C} \int_{\mathcal{H}^{2}} \int_{\mathcal{H}^{2}} R_{\mathrm{ir}}\left(\boldsymbol{x}, \omega_{i}, \omega_{o}\right) \mathrm{d} \omega_{i} \mathrm{~d} \omega_{o}
$$

where

$$
C=\int_{\mathcal{P}_{\text {all }}} \int_{\mathcal{H}^{2}} \int_{\mathcal{H}^{2}} R_{\mathrm{ir}}\left(\boldsymbol{x}_{m}(\boldsymbol{p}), \boldsymbol{\omega}_{i}, \boldsymbol{\omega}_{o}\right) k_{\mathcal{P}_{\text {all }}}(\boldsymbol{p}) \mathrm{d} \omega_{i} \mathrm{~d} \omega_{o} \mathrm{~d} \boldsymbol{p},
$$

is a normalization factor, and $\mathcal{P}_{\text {all }}=[0,1]^{2}$ covers the whole displacement map. To obtain $T_{\text {ir }}$ as a tabulated function, we estimate its value at each bin using Monte Carlo integration based on a sparse (joint) sampling of $x$ (among all locations within the bin), $\omega_{i}$, and $\omega_{o}$. We provide more implementation details in $§ 7$.

On the other hand, the angular scaling function $S_{\mathrm{ir}}\left(\omega_{i}, \omega_{o}\right)$ can be obtained by averaging $R_{\text {ir }}$ spatially:

$$
S_{\mathrm{ir}}\left(\boldsymbol{\omega}_{i}, \omega_{o}\right)=\int_{\mathcal{P}_{\text {all }}} R_{\mathrm{ir}}\left(\boldsymbol{x}_{m}(\boldsymbol{p}), \boldsymbol{\omega}_{i}, \omega_{o}\right) k_{\mathcal{P}_{\text {all }}}(\boldsymbol{p}) \mathrm{d} \boldsymbol{p} .
$$

As a tabulated function, $S_{\text {ir }}$ can be computed in a similar way as $T_{\text {ir }}$ using Monte Carlo integration.

It is easy to verify that our definitions of $T_{\text {ir }}$ and $S_{\text {ir }}$ in Eqs. $(22,24)$ are consistent with Eq. (21). Please see Appendix B for more details.

Figure 10 compares factorization results obtained using our method and SVD (that uses full $R_{\text {ir }}$ ). Our method offers similar reconstruction errors as SVD does, which is demonstrated in Figure 10(b, c). Further, the corresponding renderings shown in Figure 10(d-f) indicate that the loss in visual quality caused by our method is negligible.

\section{IMPLEMENTATION}

We now provide details of our implementation. First, we provide details on how our technique works at individual scales (§7.1). Then, we describe how mipmaps of our prefiltered models can be created, enabling anti-aliased level-of-detail (LoD) rendering of displacement-mapped models (\$7.2).

\subsection{Prefiltering at a Single Scale}

Provided a high-resolution displacement map $h_{\text {orig }}$ and a downsampling scale, we first optimize for the downsampled displacement map using least-squares ( $\$ 4.1)$ and compute the initial spatially varying multi-lobe BRDF $f_{\text {low }}^{\prime}$ (§4.2). As in prior work [Han et al. 2007], we use six vMF lobes per patch NDF. Then, we seek the scaling function $R_{\text {ir }}$ so that the final SVBRDF $f_{\text {low }}=R_{\text {ir }} \cdot f_{\text {low }}^{\prime}$ preserves the appearance of the input model ( $\$ 4.3$ and $\S 5.2)$. This is achieved using our efficient factorization of $R_{\mathrm{ir}}(\S 6)$.

To estimate the value of $T_{\text {ir }}$ at each tabulation bin, we use 5000 pairs of $\left(\omega_{i}, \omega_{o}\right)$ with both directions independently sampled from a cosine-weighted distribution. For evaluating the scaling function $R_{\text {ir }}$ via Eq. (20), we trace 2500 paths to estimate the effective BRDFs (17). Lastly, we normalize $T_{\text {ir }}$ such that it averages to one. To estimate the scaling function $S_{\text {ir }}$ of directions, we use 16 stratified samples for $\boldsymbol{p}$ and 2500 light transport paths for $R_{\mathrm{ir}}$. We find these sampling rates are sufficient to reproduce accurate appearances.

We implement our method based on the Mitsuba physically based renderer [Jakob 2010]. Running on a workstation equipped with a six-core Intel i7-5930K CPU, computing the factorized functions $T_{\mathrm{ir}}$ and $S_{\text {ir }}$ takes 20-30 minutes for each input model.

Shell mapping. We use shell mapping [Porumbescu et al. 2005] to deform a flat displacement map to a general shape (Figure 11), instead of explicitly displacing a base mesh. In fact, objects in our scenes are perturbed by extreme high-resolution displacement maps (resolution up to $(200 K)^{2}$, tiled by $(1 K)^{2}$ base displacement maps) to describe very small geometric details. Creating an explicit displacement mesh is not practical.

Importance sampling. Our final prefiltered SVBRDF $f_{\text {low }}$ equals to the product of two functions $R_{\mathrm{ir}}$ and $f_{\text {low }}^{\prime}$. Due to the smoothness of the scaling function $R_{\text {ir }}$, we simply follow $f_{\text {low }}^{\prime}$ when importance sampling $f_{\text {low }}$. That said, product importance sampling [Clarberg et al. 2005; Herholz et al. 2016] could be used to further improve the sampling quality.

\subsection{Level of Detail}

To enable anti-aliased rendering at multiple scales, we prefilter the input model at several downsampling scales. The resulting models effectively form a mipmap. At each mipmap level, we compute and store the lobe parameters as well as the scaling functions $T_{\text {ir }}$ and $S_{\text {ir }}$, in addition to the downsampled displacement map. To balance computational and storage overhead and rendering performance, our mipmap uses a sparser set of levels compared to conventional texture mipmaps. In other words, the scales of two contiguous levels of our mipmaps usually differ by more than $2 \times$. For example, the mipmap of the "silk" model includes $(16 \times)^{2}$ - and $(64 \times)^{2}$-downsampled versions (besides the original model), and the mipmap of "twill" has $(8 \times)^{2}$ and $(64 \times)^{2}$ downsampled versions.

At render time, we use ray differentials [Igehy 1999] to compute each pixel's footprint on the original displacement map and determine the desired mipmap levels. To interpolate between two levels, we trace paths on two different models and linearly interpolate the path contributions. Please refer to Figures 1, 12 and the accompanying video for results rendered with this method.

\section{RESULTS}

We now show results generated using our method: $§ 8.1$ contains experimental evaluations and justifications; §8.2 demonstrates the 
(a) Reference

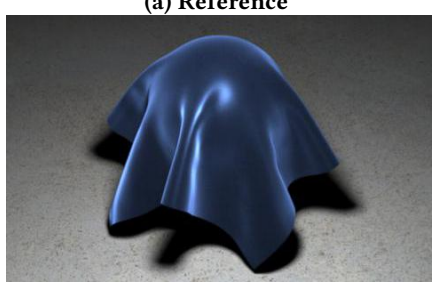

$\operatorname{MSE}\left(\times 10^{-4}\right)$ :

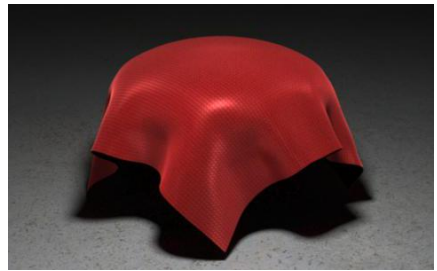

$\operatorname{MSE}\left(\times 10^{-4}\right)$ (b) $N=5$

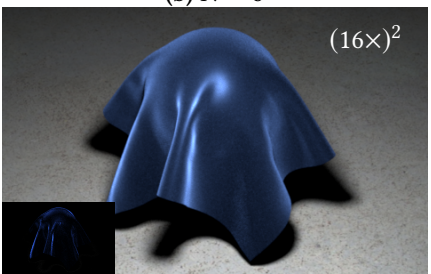

2.112

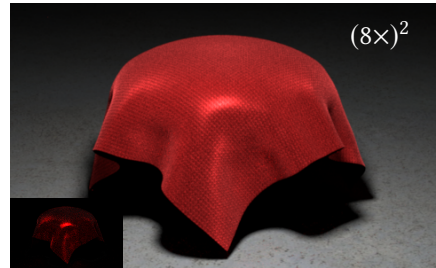

6.739 (c) $N=15$

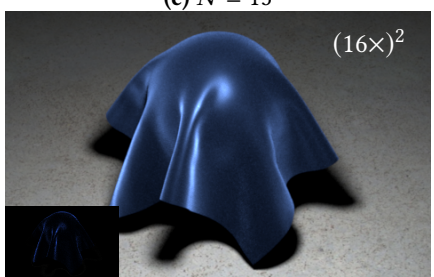

0.867

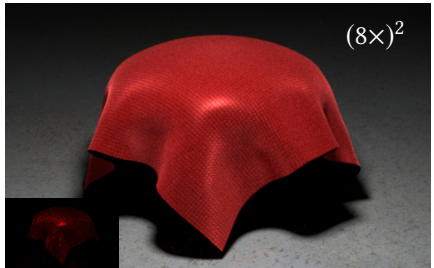

2.835 (d) $N=25$

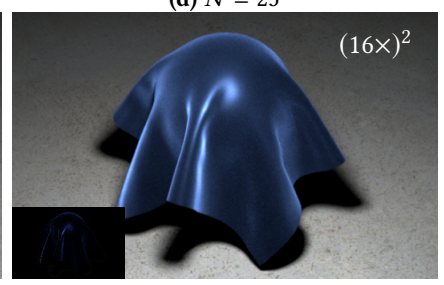

0.689

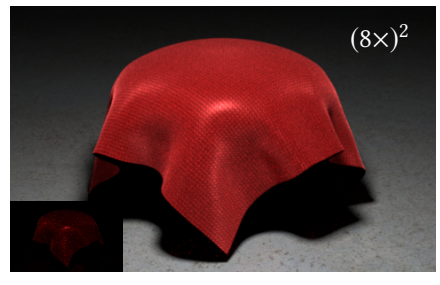

2.216

Fig. 8. Using varying angular resolutions. Increasing angular resolution improves rendering accuracy, but at the expense of larger computational and storage overhead (with a fixed spatial resolution of $M=1$ ). We use a resolution of $N=15$, which shows the best tradeoff between accuracy and storage/performance. The insets (and those in the next figures $9,10(\mathrm{e}, \mathrm{f})$ ) show $10 \times$ squared errors compared to the reference images.

(a) Reference

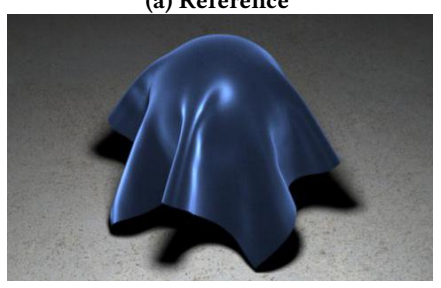

$\operatorname{MSE}\left(\times 10^{-4}\right)$

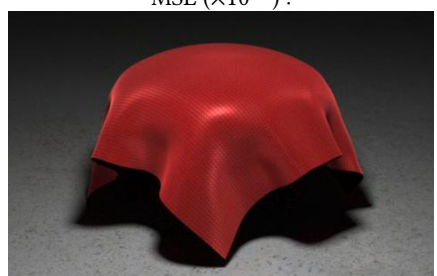

$\operatorname{MSE}\left(\times 10^{-4}\right)$ (b) $M=1$

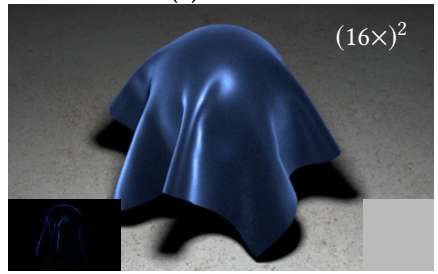

0.867

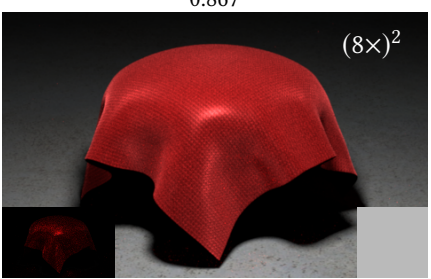

2.835 (c) $M=4$

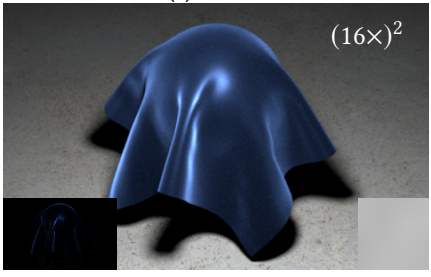

0.731

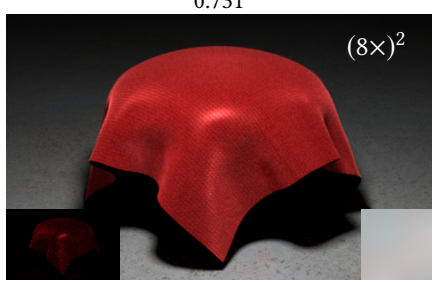

2.562 (d) $M=16$

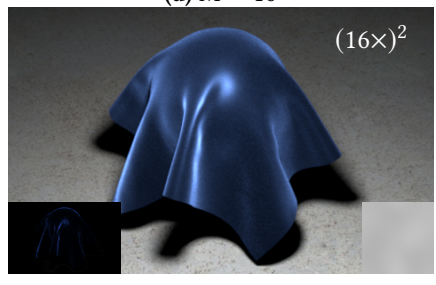

0.755

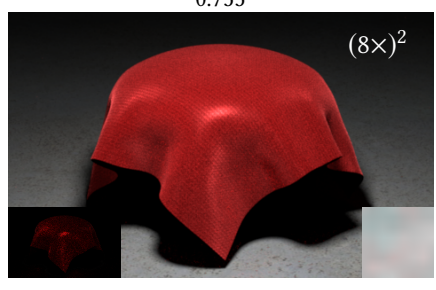

2.264

Fig. 9. Using varying spatial resolutions. Increasing spatial resolutions results in more accurate rendering results, but the improvement is diminishing after $M=4$ (errors may become slightly larger because of Monte Carlo noise). We find that $M=4$ is adequate in most cases, and even using $M=1$ yields high-quality results. Bottom-right insets visualize the 2D spatial scaling functions for different resolutions.

effectiveness of our technique via a few examples. Finally, we discuss limitations of our methods and future research directions (§8.3).

\subsection{Evaluations and Justifications}

Energy Conservation. We demonstrate that our prefiltered models conserve energy via white furnace tests (Figure 13). Given a non-absorptive base BRDF and a constant environment light, our prefiltered models without interreflections (Figure 13(b)) lose a significant amount of energy, while those capturing interreflections (Figure 13(c)) preserve most of the energy. The effective albedos of our prefiltered models in Figure 13(c) are 0.988 (twill) and 0.999 (noise).
The ground truth effective BRDFs are energy conserving as they are determined by explicitly simulating light transport within the micro-surfaces. Theoretically, our prefiltered models as approximations of the ground truth effective BRDFs are not guaranteed to conserve energy. Limited energy loss is mainly due to neglecting distant interreflections. In practice, however, we do not observe any problem with energy conservation.

Reciprocity. Our effective BRDFs (17) are reciprocal when weighted by visible projected area (similar to the microflake reciprocity constraints proposed by Heitz et al. [2015]):

$$
f_{\mathrm{ir}}^{\mathrm{eff}}\left(\omega_{i}, \omega_{o} ; \mathcal{G}, f\right) A_{\mathcal{G}}\left(\omega_{o}\right)=f_{\mathrm{ir}}^{\mathrm{eff}}\left(\omega_{o}, \omega_{i} ; \mathcal{G}, f\right) A_{\mathcal{G}}\left(\omega_{i}\right)
$$



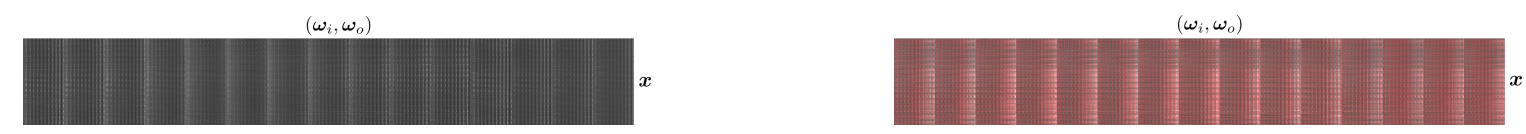

(a) Reference scaling matrix
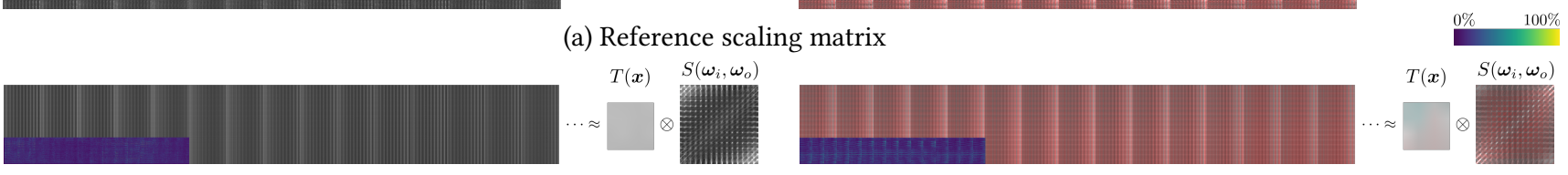

(b) Our factorization
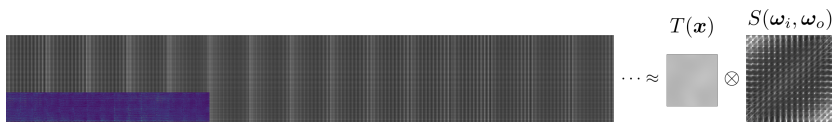

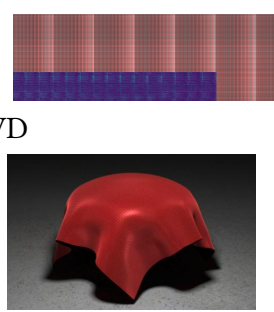

(d2) Reference

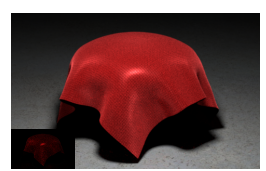

(e2) Our factorization

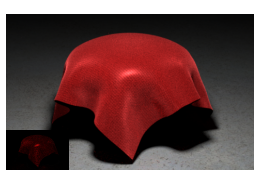

(f2) Rank-1 SVD

(d1) Reference

(e1) Our factorization

(f1) Rank-1 SVD

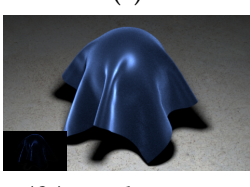

Fig. 10. Factorization comparison. We compare our factorization method to rank-1 SVD in terms of scaling function reconstruction (a-c) and rendered
images (d-f). We show 6D scaling functions (they are reorganized as scaling matrices) in (a). Even without explicitly computing the full 6D functions, the reconstruction error of our factorization (b) is similar to the error given by rank-1 SVD (c). Both errors are small because of the low-rank nature of the scaling matrices. The insets in (b, c) show the relative error maps of the reconstructed matrices compared to the reference matrices. The colored error bar is shown on the top-right. In terms of visual quality, using the factorized spatial scaling function $T$ and angular scaling function $S$ computed by our method reproduces the reference appearance accurately.

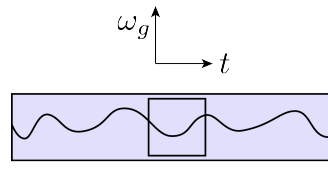

texture space
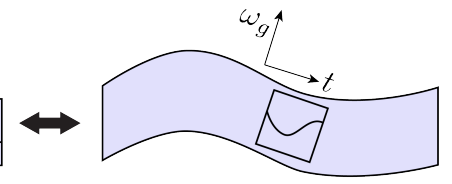

shell map
Fig. 11. Shell map illustration. We deform a flat displacement map to a general shape using shell maps.

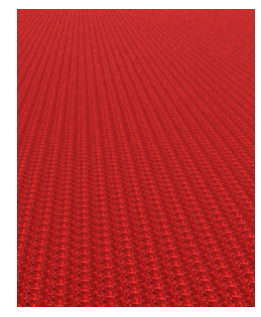

(a) Reference Noise level:

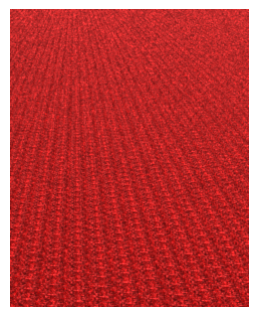

(b) Original 0.0162

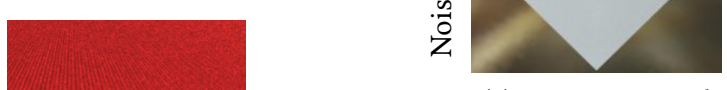

(a) Environmental lighting

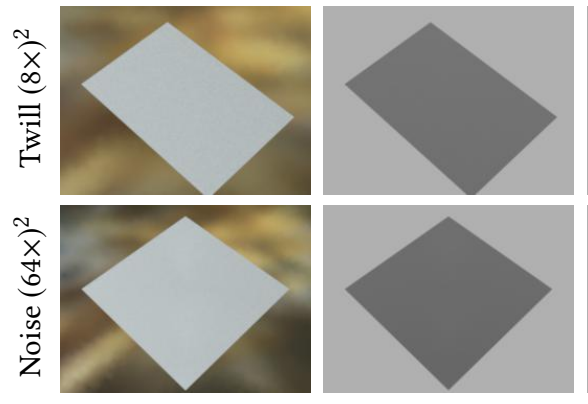

(b) Constant lighting with $R$

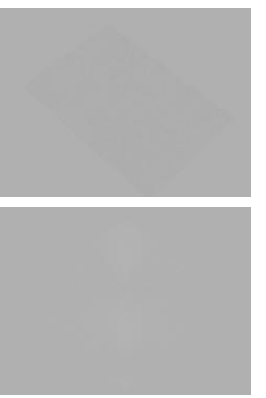

(c) Constant lighting with $R_{\text {ir }}$
Fig. 13. White furnace test. We prefilter displacement maps with a nonabsorptive BRDF. Using the scaling function $R$ handling only direct illumination (i.e., single bounce) leads to significant energy loss. Taking interreflections into account, the object rendered with the multi-bounce scaling function $R_{\text {ir }}$ becomes almost invisible, showing negligible energy loss.

example where the surface normals and colors are strongly correlated. The baseline multi-lobe BRDFs $f_{\text {low }}^{\prime}$ disregard this correlation and produce wrong appearance. Our result, on the other hand, captures this correlation properly and preserves the reference appearance accurately.

\subsection{Main Results}

We use two sets of scenes to demonstrate the effectiveness of our method. The scene objects and their detailed parameters (before and after prefiltering) are summarized in Table 3 . The first set contains two fabric examples (Figures 2, 16, 17). The "twill" and "silk" models 


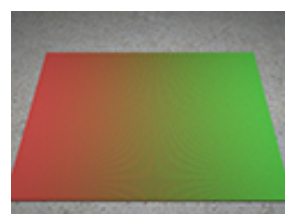

(a) Reference

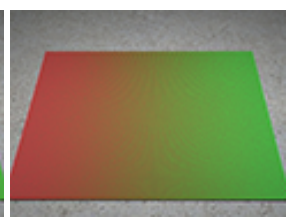

(b) Ours $\left(R_{\mathrm{ir}} \cdot f_{\text {low }}^{\prime}\right)$

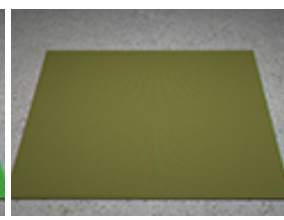

(c) Multi-Lobe $\left(f_{\text {low }}^{\prime}\right)$
Fig. 14. Correlated surface. We prefilter a two-color sawtooth (i.e., Vgroove) surface. Thanks to the scaling function $R_{\mathrm{ir}}$, our prefiltered model can effectively capture the normal-color correlation and accurately reproduce the appearance.

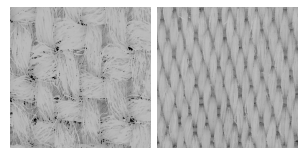

(a) Twill (b) Silk

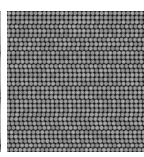

(c) Bump

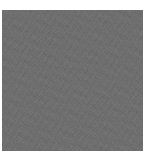

(d) Noise

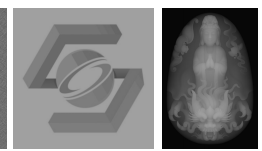

(e) Logo (f) Relief
Fig. 15. Displacement maps used in our results.

(Figure 15(a, b)) are extracted from detailed volume data [Zhao et al. 2011]. The resulting surface micro-geometries are highly complex and can cause severe aliasing. The "plane" scene in Figure 2 contains a flat twill fabric. The "twill" scene in Figures 16, 17 (top row) uses the same fabric but shell-mapped. The "silk" scene in Figures 16, 17 (bottom row) has another shell-mapped silk fabric. Although we use isotropic glossy BRDFs in these scenes, the rendered images still show anisotropic glossy highlights because of the complex structured micro-geometries.

Another set of scenes contains objects with more diverse materials. In Figure 18 (top row), we show the "ball" scene that has a bumpy ball on a rough floor. The ball is represented by the "bump" model (Figure 15(c)), while the floor is represented by the "noise" model (Figure 15(d)). Both models use diffuse base BRDFs. In Figure 18 (middle row), we show the "logo" scene that is represented by a biscale displacement map (Figure 15(e)). The large-scale displacement map describes the overall shape, while the small-scale displacement map (which is a tiled version of the large-scale one) accounts for the detailed micro-geometry. In Figure 18 (bottom row), we show the "relief" scene that is represented by a composite displacement map (Figure 15(f), which is resampled to keep the image aspect ratio). It is constructed by adding procedural Perlin noise on a height field converted from a gray scale image. In this example, we use a spatial scaling function with resolution $M=1$ since it leads to sufficient accuracy.

Direct Illumination. Figure 16 contains rendered images generated through the intermediate steps of our method with the effective BRDFs capturing only direct illumination (i.e., neglecting micro-scale interreflections). Directly using original BRDFs $f_{\text {orig }}$ with downsampled micro-geometries (the third column) leads to significantly different appearances. Using the spatially varying multilobe BRDF $f_{\text {low }}^{\prime}$ without our scaling function $R=S T$ (the fourth column) performs better, but the results are still notably brighter than the references as changes of shadowing and masking are neglected. By applying a further correction using $R=S T$, our models well preserve the input appearances. Additionally, we compare our prefiltered models with those generated using LEADR [Dupuy et al.
2013], which are derived from single-lobe Beckmann NDFs and Smith's shadowing function. These models fail to reproduce the appearance of the input since the assumption of Gaussian surfaces does not hold (see the top insets in Figure $2(a, f)$ ). Further, the single lobe representation used by LEADR also limits the result accuracy, especially for glossy base BRDFs.

Global Illumination. The results in Figures 17 and 18 are rendered with full global illumination. In this case, directly using $f_{\text {orig }}$ (the third column) still yields poor accuracy. Using multi-lobe BRDFs $f_{\text {low }}^{\prime}$ improves the glossy highlights quality but cannot fully capture the change of interreflections. Our full model with $f_{\text {low }}^{\prime}$ and the scaling functions $R_{\mathrm{ir}}=S_{\mathrm{ir}} T_{\mathrm{ir}}$ resembles the input appearance accurately. To demonstrate the effectiveness and generality of our technique, we also show rendering results under different lighting and viewing configurations in Figure 17 and the accompanying video.

Performance and Storage. Table 3 summarizes the storage size and precomputation time of our prefiltered models and Table 4 shows equal-time mean squared error (MSE) comparisons. Our prefiltered models can offer significant storage reduction at a single downsampling scale. Breaking down individual components of our model, the multi-lobe SVNDFs are most storage-consuming (except "noise" and "logo" as they are greatly downsampled) while our scaling functions $T$ and $S$ only introduce minimal overhead.

Our model also benefits rendering performance by reducing ray tracing cost. We demonstrate this by rendering a few highresolution models and their prefiltered counterparts (generated with our method) in equal-time and compare MSEs of the results (see Table 4). Using our prefiltered models leads to lower errors in all the scenes and anti-aliased renderings with better visual qualities (see Figure 2(c, e)). In the accompanying video, we demonstrate that our prefiltered models preserve input appearance accurately and reduce flickering significantly.

\subsection{Limitations and Future Work}

Figure 19 shows a failure example where our method does not accurately reproduce the original appearance caused by interreflections. This "statue" scene contains a $(4 K)^{2}$ displacement map and a moderately glossy base BRDF. We downsample it by $(16 \times)^{2}$ and use a spatial scaling function of resolution $M=1$. Compared to the "relief" scene, the vertical displacements in this scene are much greater. In this case, distant interreflections can only be partially handled by explicit path tracing. Since our method neglects these interreflections (for enabling efficient computation of $S_{\mathrm{ir}}$ and $T_{\mathrm{ir}}$ ), the results have visible accuracy loss shown as darkening at the steep edges (see (b1) and (b2)). On the other hand, although our method produces inaccurate edge appearance, the overall surface reflectance (i.e. average image intensity) still matches the reference because the two effective BRDFs are matched by our scaling functions. For direct illumination results ((a1) and (a2)), the accuracy is not affected.

Since our method considers micro-geometries and base BRDFs jointly, which is necessary for accurately handling complex light transport effects such as shadowing-masking and interreflections, changing the micro-geometry or base BRDF requires recomputing our prefiltered models. In the future, our technique may be 

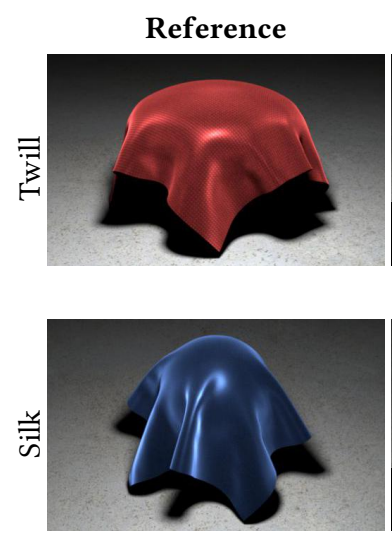
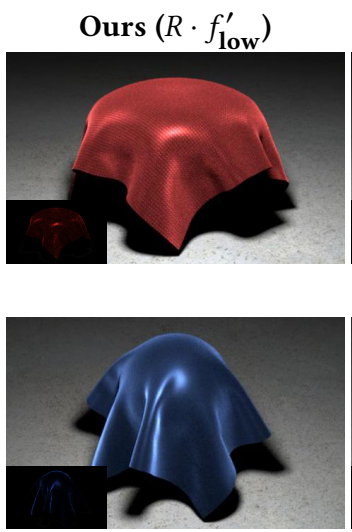

Naïve ( $\left.f_{\text {orig }}\right)$

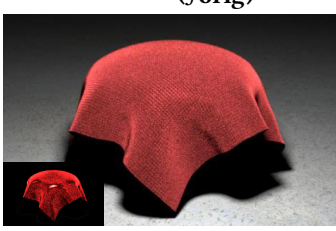

$(8 \times)^{2}$-downsampled.

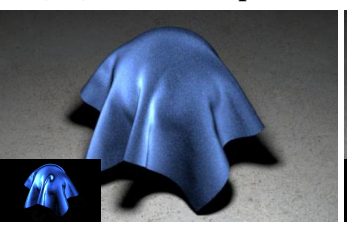

$(16 \times)^{2}$-downsampled.
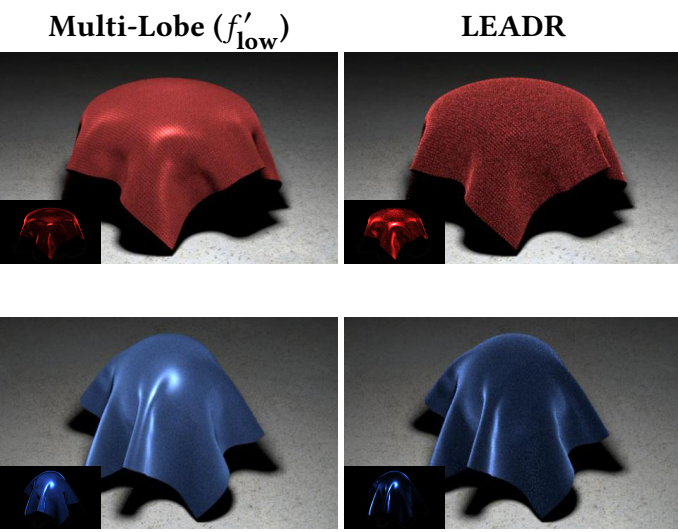

Fig. 16. Direct illumination results. Our method accurately matches the reference even with significant downsampling, and is much more accurate than naive downsampling, multi-lobe BRDFs without taking shadowing-masking or interreflections into account, and LEADR. The insets (and those in the next figures 17,18$)$ show $10 \times$ squared errors compared to the reference images.
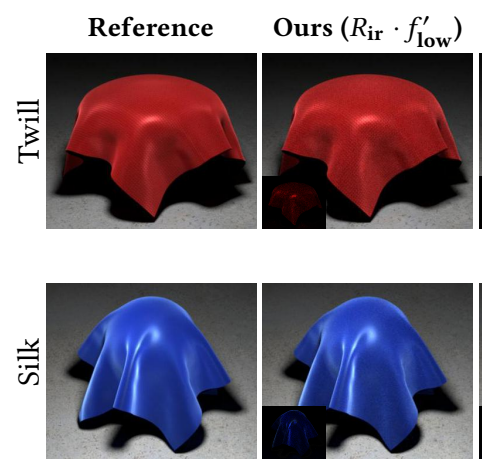
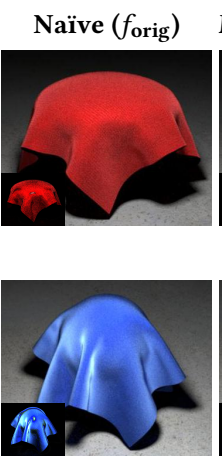
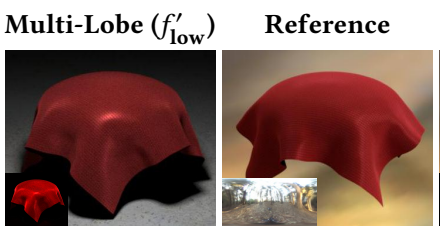

$(8 \times)^{2}$-downsampled.

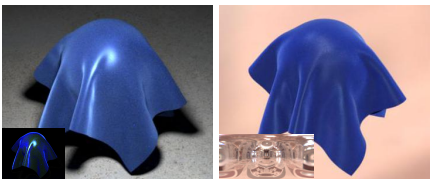

$(16 \times)^{2}$-downsampled.
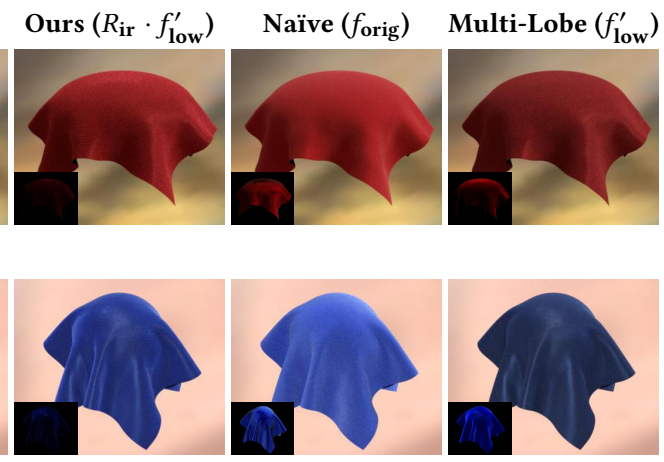

Fig. 17. Global illumination results. The four columns on the left are rendered under local area lights, while the four columns on the right are rendered under environmental lightings (environment maps are visualized as insets of the reference images) from a different view. Our method is able to capture interreflections well, closely matching the reference. Naive downsampling and the multi-lobe BRDFs do not model interreflections and have significant errors, while LEADR does not perform well even for direct illumination and is not designed for interreflections (and is therefore not shown here).

Table 3. Model Information. We list the detailed parameters used for prefiltering the input model at a single downsampling scale. We also provide the data size of our prefiltered models and their corresponding precomputation time.

\begin{tabular}{|c|c|c|c|c|c|c|c|c|c|c|c|c|c|c|}
\hline & \multicolumn{4}{|c|}{ Original model } & \multicolumn{6}{|c|}{ Our prefiltered model } & \multicolumn{4}{|c|}{ Precomputation time } \\
\hline Name & Resolution & Tiling & $\begin{array}{c}\text { Total size } \\
(\mathrm{KB})\end{array}$ & $\begin{array}{c}\text { Base } \\
\text { BRDF }\end{array}$ & $\begin{array}{c}\text { Downsampling } \\
\text { scale }\end{array}$ & $\begin{array}{c}\text { Total size } \\
(\mathrm{KB})\end{array}$ & $\begin{array}{l}h_{\text {low }} \\
(\mathrm{KB})\end{array}$ & $\begin{array}{l}f_{\text {low }}^{\prime} \\
(\mathrm{KB})\end{array}$ & $\begin{array}{l}T / T_{\text {ir }} \\
(\mathrm{KB})\end{array}$ & $\begin{array}{l}S / S_{\text {ir }} \\
(\mathrm{KB})\end{array}$ & $\begin{array}{c}\text { Total time } \\
(\mathrm{min})\end{array}$ & $\begin{array}{c}h_{\text {low }} \text { and } \\
f_{\text {low }}^{\prime}(\min )\end{array}$ & $\begin{array}{l}T / T_{\mathrm{ir}} \\
(\mathrm{min})\end{array}$ & $\begin{array}{l}S / S_{\text {ir }} \\
(\mathrm{min})\end{array}$ \\
\hline Twill & $(1 K)^{2}$ & $100^{2}$ & 3502 & glossy & $(8 x)^{2}$ & 2377 & 65 & 1915 & 1 & 396 & 22 & 1 & 7 & 14 \\
\hline Silk & $(1 K)^{2}$ & $200^{2}$ & 2724 & glossy & $(16 \times)^{2}$ & 1085 & 17 & 478 & 1 & 589 & 22 & 1 & 7 & 14 \\
\hline Bump & $(1 K)^{2}$ & $4^{2}$ & 4015 & diffuse & $(16 \times)^{2}$ & 704 & 17 & 484 & 1 & 202 & 24 & 1 & 3 & 20 \\
\hline Noise & $(1 K)^{2}$ & $100^{2}$ & 3954 & diffuse & $(64 \times)^{2}$ & 239 & 2 & 34 & 1 & 202 & 22 & 1 & 4 & 17 \\
\hline $\log 0^{6}$ & $(1 K)^{2}$ & $(1 K)^{2}$ & 723 & glossy & $(1 K \times)^{2}$ & 589 & $\sim 0$ & 2 & 1 & 586 & 36 & 1 & 12 & 23 \\
\hline Relief & $(4 K)^{2}$ & $1^{2}$ & 33114 & glossy & $(16 \times)^{2}$ & 8367 & 238 & 7619 & $0(\mathrm{M}=1)$ & 510 & 26 & 1 & 0 & 25 \\
\hline
\end{tabular}

combined with material editing techniques such as Hašan and Ramamoorthi [2013] to allow changing the base BRDF with a single precomputation.

\footnotetext{
${ }^{6}$ We provide only parameters of the small-scale displacement map, since the largescale one remains unchanged when downsampling.
}

Interreflection is an effect that is well known to be challenging to analyze due to its high nonlinearity. A theoretical or data-driven analysis can be an interesting topic to explore. To balance accuracy and efficiency, we use relatively low resolutions for the spatial and 
Table 4. Equal-time performance. Our prefiltered models not only reduce the storage size, but also bring benefits in rendering. Given equal time (around 60s for 50-100 spp), rendering using our models results in anti-aliased images, which have lower MSE than those rendered using the original models.

\begin{tabular}{|c|c|c|c|c|c|}
\hline Scene & Twill & Silk & Ball & Logo & Relief \\
\hline Original model's MSE $\left(\times 10^{-3}\right)$ & 1.095 & 1.269 & 2.055 & 2.072 & 2.946 \\
\hline Our model's MSE $\left(\times 10^{-3}\right)$ & 0.571 & 0.622 & 1.850 & 0.498 & 1.692 \\
\hline
\end{tabular}
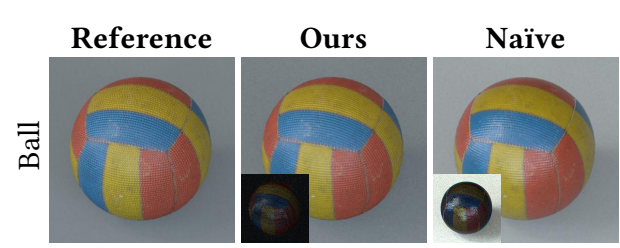

Multi-Lobe

Ball: $(16 \times)^{2}$-downsampled. Floor: $(64 \times)^{2}$-downsampled.
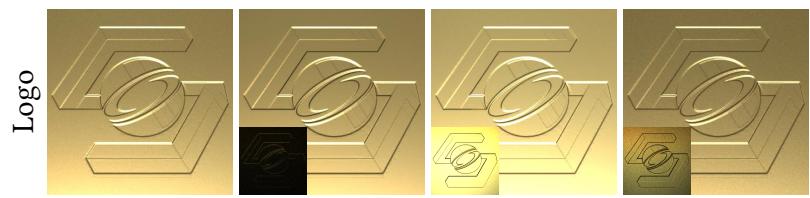

$(1 K \times)^{2}$-downsampled.
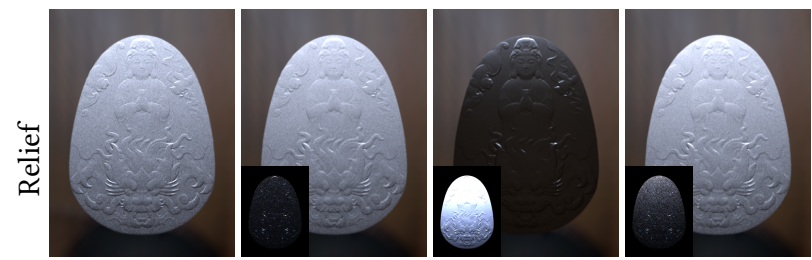

$(16 \times)^{2}$-downsampled.

Fig. 18. Global illumination results. We show results of more materials. Images rendered using our prefiltered models best match the references.

angular scaling functions empirically. We leave how to determine the optimal resolutions as future work.

We assume the base BRDFs to be isotropic because the anisotropic micro-geometries are capable of reproducing anisotropic appearance. Using anisotropic base BRDFs would increase the model expressiveness but at the price of more expensive precomputation. We leave this extension for future exploration.

Additionally, as our technique only introduces minimal storage overhead, it may benefit real-time and interactive rendering applications.

\section{CONCLUSION}

We introduce a novel method to prefilter the surface reflectance generated by high-resolution displacement maps while accurately preserving the input appearance. Our prefiltered models leverage SVBRDFs expressed by a SVNDF coupled with a 6D scaling function that captures the change of shadowing-masking and interreflection effects caused by the downsampling of micro-geometries. We further introduce an efficient approach to factorize this $6 \mathrm{D}$ function into a 2D function of location and a 4D function of direction. Our models

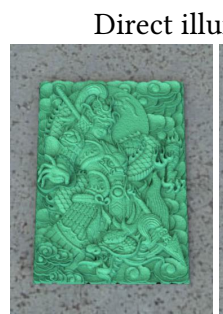

(a1) Reference

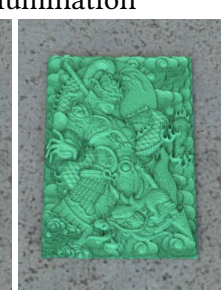

(a2) Ours

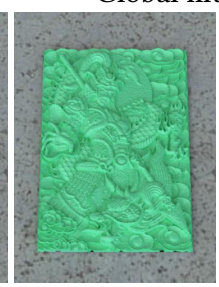

(b1) Reference
Global illumination

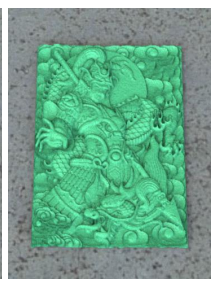

(b2) Ours
Fig. 19. Failure case. Our method accurately handles direct illumination, but is unable to match the appearance with global illumination, since distant interreflections are not handled with sufficient accuracy.

are capable of producing accurate appearance that matches the original one. In practice, our prefiltered models offer significant storage reduction and efficient anti-aliased rendering. Additionally, multiple models generated using our technique with varying downsampling scales can be combined to form a mipmap, allowing level-of-detail rendering of detailed surfaces in an efficient and consistent manner.

\section{ACKNOWLEDGMENTS}

We thank the anonymous reviewers for their constructive suggestions and comments. This work was supported in part by NSF grants 1451830, 1703957, and 1813553, an NVIDIA fellowship, the Ronald L. Graham Chair, and the UC San Diego Center for Visual Computing.

\section{REFERENCES}

Michael Ashikmin, Simon Premože, and Peter Shirley. 2000. A microfacet-based BRDF generator. SIGGRAPH (2000), 65-74.

Eric Bruneton and Fabrice Neyret. 2012. A survey of nonlinear prefiltering methods for efficient and accurate surface shading. IEEE Transactions on Visualization and Computer Graphics 18, 2 (2012), 242-260.

Brian Cabral, Nelson Max, and Rebecca Springmeyer. 1987. Bidirectional reflection functions from surface bump maps. SIGGRAPH (1987), 273-281.

Xavier Chermain, Frédéric Claux, and Stéphane Mérillou. 2018. A microfacet-based BRDF for the accurate and efficient rendering of high-definition specular normal maps. The Visual Computer (2018)

Petrik Clarberg, Wojciech Jarosz, Tomas Akenine-Möller, and Henrik Wann Jensen. 2005. Wavelet Importance Sampling: Efficiently Evaluating Products of Complex Functions. ACM Trans. Graph. 24, 3 (2005), 1166-1175.

Robert L Cook and Kenneth E. Torrance. 1982. A reflectance model for computer graphics. SIGGRAPH (1982), 7-24.

Kristin J Dana, Bram Van Ginneken, Shree K Nayar, and Jan J Koenderink. 1999. Reflectance and texture of real-world surfaces. ACM Trans. Graph. 18, 1 (1999), 1-34.

Jonathan Dupuy, Eric Heitz, Jean-Claude Iehl, Pierre Poulin, Fabrice Neyret, and Victor Ostromoukhov. 2013. Linear efficient antialiased displacement and reflectance mapping. ACM Trans. Graph. 32, 6 (2013), 211:1-211:11.

Alain Fournier. 1992. Normal Distribution Functions and Multiple Surfaces. (1992).

Ioannis Gkioulekas, Anat Levin, and Todd Zickler. 2016. An evaluation of computational imaging techniques for heterogeneous inverse scattering. In European Conference on Computer Vision. 685-701.

Ioannis Gkioulekas, Shuang Zhao, Kavita Bala, Todd Zickler, and Anat Levin. 2013 Inverse Volume Rendering with Material Dictionaries. ACM Trans. Graph. 32, 6 (2013), 162:1-162:13.

Charles Han, Bo Sun, Ravi Ramamoorthi, and Eitan Grinspun. 2007. Frequency Domain Normal Map Filtering. ACM Trans. Graph. 26, 3 (2007), 28:1-28:12.

Milovš Hašan and Ravi Ramamoorthi. 2013. Interactive Albedo Editing in Path-traced Volumetric Materials. ACM Trans. Graph. 32, 2 (2013), 11:1-11:11.

Wolfgang Heidrich, Katja Daubert, Jan Kautz, and Hans-Peter Seidel. 2000. Illuminating micro geometry based on precomputed visibility. SIGGRAPH (2000), 455-464.

Eric Heitz. 2014. Understanding the masking-shadowing function in microfacet-based BRDFs. Journal of Computer Graphics Techniques 3, 2 (2014), 32-91.

Eric Heitz, Jonathan Dupuy, Cyril Crassin, and Carsten Dachsbacher. 2015. The SGGX Microflake Distribution. ACM Trans. Graph. 34, 4 (2015), 48:1-48:11.

Eric Heitz, Johannes Hanika, Eugene d'Eon, and Carsten Dachsbacher. 2016. Multiplescattering microfacet BSDFs with the Smith model. ACM Trans. Graph. 35, 4 (2016), 
58:1-58:14.

Sebastian Herholz, Oskar Elek, Jiř́ Vorba, Hendrik Lensch, and Jaroslav Křivánek. 2016 Product importance sampling for light transport path guiding. Computer Graphics Forum 35, 4 (2016), 67-77.

Homan Igehy. 1999. Tracing Ray Differentials. SIGGRAPH (1999), 179-186.

Kei Iwasaki, Yoshinori Dobashi, and Tomoyuki Nishita. 2012. Interactive bi-scale editing of highly glossy materials. ACM Trans. Graph. 31, 6 (2012), 144:1-144:7.

Wenzel Jakob. 2010. Mitsuba renderer. http://www.mitsuba-renderer.org.

Wenzel Jakob, Miloš Hašan, Ling-Qi Yan, Jason Lawrence, Ravi Ramamoorthi, and Steve Marschner. 2014. Discrete Stochastic Microfacet Models. ACM Trans. Graph. 33, 4 (2014), 115:1-115:10

Adrian Jarabo, Hongzhi Wu, Julie Dorsey, Holly Rushmeier, and Diego Gutierrez. 2014. Effects of Approximate Filtering on the Appearance of Bidirectional Texture Functions. IEEE Transactions on Visualization and Computer Graphics (2014), 880892.

Anton S Kaplanyan, Stephen Hill, Anjul Patney, and Aaron E Lefohn. 2016. Filtering distributions of normals for shading antialiasing.. In High Performance Graphics. 151-162.

Pramook Khungurn, Daniel Schroeder, Shuang Zhao, Kavita Bala, and Steve Marschner. 2015. Matching Real Fabrics with Micro-Appearance Models. ACM Trans. Graph. 35,1 (2015), 1:1-1:26.

Joo Ho Lee, Adrian Jarabo, Daniel S. Jeon, Diego Gutierrez, and Min H. Kim. 2018 Practical Multiple Scattering for Rough Surfaces. ACM Trans. Graph. 37, 6 (2018), 275:1-275:12

Xinhao Liu, Mitsuru Tanaka, and Masatoshi Okutomi. 2012. Noise level estimation using weak textured patches of a single noisy image. In IEEE International Conference on Image Processing (ICIP) 2012. 665-668.

Guillaume Loubet and Fabrice Neyret. 2017. Hybrid mesh-volume LoDs for all-scale pre-filtering of complex 3D assets. Computer Graphics Forum 36, 2 (2017), 431-442.

Wan-Chun Ma, Sung-Hsiang Chao, Yu-Ting Tseng, Yung-Yu Chuang, Chun-Fa Chang, Bing-Yu Chen, and Ming Ouhyoung. 2005. Level-of-detail representation of bidirectional texture functions for real-time rendering. Proceedings of the 2005 symposium on Interactive 3D graphics and games (2005), 187-194.

Derek Nowrouzezahrai and John Snyder. 2009. Fast global illumination on dynamic height fields. Computer Graphics Forum 28, 4 (2009), 1131-1139.

Marc Olano and Dan Baker. 2010. LEAN mapping. Proceedings of the 2010 ACM SIGGRAPH symposium on Interactive 3D Graphics and Games (2010), 181-188.

Michael Oren and Shree K Nayar. 1994. Generalization of Lambert's reflectance model. SIGGRAPH (1994), 239-246

Serban D Porumbescu, Brian Budge, Louis Feng, and Kenneth I Joy. 2005. Shell maps ACM Trans. Graph. 24, 3 (2005), 626-633.

Peter Shirley and Kenneth Chiu. 1997. A low distortion map between disk and square fournal of Graphics Tools 2, 3 (1997), 45-52.

B Smith. 1967. Geometrical shadowing of a random rough surface. IEEE transactions on antennas and propagation 15, 5 (1967), 668-671.

John Snyder and Derek Nowrouzezahrai. 2008. Fast Soft Self-Shadowing on Dynamic Height Fields. Computer Graphics Forum 27, 4 (2008), 1275-1283.

Ping Tan, Stephen Lin, Long Quan, Baining Guo, and Harry Shum. 2008. Filtering and rendering of resolution-dependent reflectance models. IEEE Transactions on Visualization and Computer Graphics 14, 2 (2008), 412-425.

Ping Tan, Stephen Lin, Long Quan, Baining Guo, and Heung-Yeung Shum. 2005. Multiresolution Reflectance Filtering. Rendering Techniques (2005), 111-116.

Ville Timonen and Jan Westerholm. 2010. Scalable Height Field Self-Shadowing. Computer Graphics Forum 29, 2 (2010), 723-731.

Michael Toksvig. 2005. Mipmapping normal maps. Journal of Graphics Tools 10, 3 (2005), 65-71.

Eric Veach. 1997. Robust monte carlo methods for light transport simulation. Stanford University PhD thesis (1997).

Bruce Walter, Stephen R Marschner, Hongsong Li, and Kenneth E Torrance. 2007. Microfacet models for refraction through rough surfaces. Proceedings of the 18th Eurographics conference on Rendering Techniques, 195-206.

Stephen H. Westin, James R. Arvo, and Kenneth E. Torrance. 1992. Predicting Reflectance Functions from Complex Surfaces. SIGGRAPH (1992), 255-264.

Hongzhi Wu, Julie Dorsey, and Holly Rushmeier. 2009. Characteristic point maps. Computer Graphics Forum 28, 4 (2009), 1227-1236.

Hongzhi Wu, Julie Dorsey, and Holly Rushmeier. 2011. Physically-based interactive bi-scale material design. ACM Trans. Graph. 30, 6 (2011), 145:1-145:10.

Feng Xie and Pat Hanrahan. 2018. Multiple Scattering from Distributions of Specular V-grooves. ACM Trans. Graph. 37, 6 (2018), 276:1-276:14.

Chao Xu, Rui Wang, Shuang Zhao, and Hujun Bao. 2017. Real-Time Linear BRDF MIP-Mapping. Computer Graphics Forum 36, 4 (2017), 27-34.

Kun Xu, Wei-Lun Sun, Zhao Dong, Dan-Yong Zhao, Run-Dong Wu, and Shi-Min Hu. 2013. Anisotropic spherical gaussians. ACM Trans. Graph. 32, 6 (2013), 209:1-209:11.

Ling-Qi Yan, Miloš Hašan, Wenzel Jakob, Jason Lawrence, Steve Marschner, and Ravi Ramamoorthi. 2014. Rendering Glints on High-Resolution Normal-Mapped Specular Surfaces. ACM Trans. Graph. 33, 4 (2014), 116:1-116:9.
Ling-Qi Yan, Miloš Hašan, Steve Marschner, and Ravi Ramamoorthi. 2016. PositionNormal Distributions for Efficient Rendering of Specular Microstructure. ACM Trans. Graph. 35, 4 (2016), 56:1-56:9.

Shuang Zhao, Wenzel Jakob, Steve Marschner, and Kavita Bala. 2011. Building Volumetric Appearance Models of Fabric Using Micro CT Imaging. ACM Trans. Graph. 30, 4 (2011), 44:1-44:10.

Shuang Zhao, Lifan Wu, Frédo Durand, and Ravi Ramamoorthi. 2016. Downsampling scattering parameters for rendering anisotropic media. ACM Trans. Graph. 35, 6 (2016), 116:1-116:9

Tobias Zirr and Anton S Kaplanyan. 2016. Real-time rendering of procedural multiscale materials. In Proceedings of the 20th ACM SIGGRAPH Symposium on Interactive $3 D$ Graphics and Games. 139-148.

\section{A AVERAGE SLOPE OF A BILINEAR PATCH}

Let $h_{00}, h_{10}, h_{01}, h_{11}$ be the height values at the four corners of a unit patch $[0,1]^{2}$. Then, the height at any point $(u, v)$ within the patch can be obtained via a bilinear interpolation:

$h(u, v)=(1-u)(1-v) h_{00}+u(1-v) h_{10}+v(1-u) h_{01}+u v h_{11}$.

The slope at each point can be computed as

$x_{s}(u, v)=\frac{h(u+\Delta u, v)-h(u, v)}{\Delta u}=h_{10}-h_{00}+v\left(h_{00}+h_{11}-h_{10}-h_{01}\right)$,

$y_{s}(u, v)=\frac{h(u, v+\Delta v)-h(u, v)}{\Delta v}=h_{01}-h_{00}+u\left(h_{00}+h_{11}-h_{10}-h_{01}\right)$

So the average slope of the bilinear patch is

$$
\begin{aligned}
& x_{\tilde{s}}=\int_{0}^{1} \int_{0}^{1} x_{s}(u, v) \mathrm{d} u \mathrm{~d} v=\frac{1}{2}\left(h_{11}+h_{10}-h_{01}-h_{00}\right), \\
& y_{\tilde{s}}=\int_{0}^{1} \int_{0}^{1} y_{s}(u, v) \mathrm{d} u \mathrm{~d} v=\frac{1}{2}\left(h_{11}+h_{01}-h_{10}-h_{00}\right) .
\end{aligned}
$$

\section{B FACTORIZATION OF $R_{\text {ir }}$}

Expanding $T_{\mathrm{ir}}(\boldsymbol{x})$ as Eqs. $(22,23)$ and $S_{\mathrm{ir}}\left(\boldsymbol{\omega}_{i}, \boldsymbol{\omega}_{\boldsymbol{o}}\right)$ as Eq. (24) gives

$$
\begin{aligned}
T_{\mathrm{ir}}(\boldsymbol{x}) S_{\mathrm{ir}}\left(\omega_{i}, \omega_{o}\right)= & \frac{\int_{\mathcal{H}^{2}} \int_{\mathcal{H}^{2}} R_{\mathrm{ir}}\left(\boldsymbol{x}, \omega_{i}, \omega_{o}\right) \mathrm{d} \omega_{i} \mathrm{~d} \omega_{o}}{\int_{\mathcal{P}_{\text {all }}} \int_{\mathcal{H}^{2}} \int_{\mathcal{H}^{2}} R_{\mathrm{ir}}\left(\boldsymbol{x}_{m}(\boldsymbol{p}), \omega_{i}, \omega_{o}\right) k_{\mathcal{P}_{\text {all }}}(\boldsymbol{p}) \mathrm{d} \omega_{i} \mathrm{~d} \omega_{o} \mathrm{~d} \boldsymbol{p}} \\
& \times \int_{\mathcal{P}_{\text {all }}} R_{\mathrm{ir}}\left(\boldsymbol{x}_{m}(\boldsymbol{p}), \omega_{i}, \omega_{o}\right) k_{\mathcal{P}_{\text {all }}}(\boldsymbol{p}) \mathrm{d} \boldsymbol{p}
\end{aligned}
$$

As $R_{\mathrm{ir}}\left(\boldsymbol{x}, \omega_{i}, \omega_{o}\right) \approx T_{\mathrm{ir}}(\boldsymbol{x}) \cdot S_{\mathrm{ir}}\left(\omega_{i}, \omega_{o}\right)$, we can simplify this formula by factoring out $R_{\mathrm{ir}}$ and regrouping the terms:

$$
\begin{aligned}
& T_{\mathrm{ir}}(\boldsymbol{x}) \int_{\mathcal{H}^{2}} \int_{\mathcal{H}^{2}} S_{\mathrm{ir}}\left(\omega_{i}, \omega_{o}\right) \mathrm{d} \omega_{i} \mathrm{~d} \omega_{o} \\
& \overline{\int_{\mathcal{P}_{\text {all }}} T_{\text {ir }}\left(\boldsymbol{x}_{m}(\boldsymbol{p})\right) k_{\mathcal{P}_{\text {all }}}(\boldsymbol{p})\left[\int_{\mathcal{H}^{2}} \int_{\mathcal{H}^{2}} S_{\text {ir }}\left(\omega_{i}, \omega_{o}\right) \mathrm{d} \omega_{i} \mathrm{~d} \omega_{o}\right] \mathrm{d} \boldsymbol{p}} \\
& \times S_{\text {ir }}\left(\omega_{i}, \omega_{o}\right) \int_{\mathcal{P}_{\text {all }}} T_{\text {ir }}\left(\boldsymbol{x}_{m}(\boldsymbol{p})\right) k_{\mathcal{P}_{\text {all }}}(\boldsymbol{p}) \mathrm{d} \boldsymbol{p} \\
& =\frac{T_{\mathrm{ir}}(\boldsymbol{x}) \int_{\mathcal{H}^{2}} \int_{\mathcal{H}^{2}} S_{\mathrm{ir}}\left(\omega_{i}, \omega_{o}\right) \mathrm{d} \omega_{i} \mathrm{~d} \omega_{o}}{\left[\int_{\mathcal{P}_{\text {all }}} T_{\mathrm{ir}}\left(\boldsymbol{x}_{m}(\boldsymbol{p})\right) k \mathcal{P}_{\text {all }}(\boldsymbol{p}) \mathrm{d} \boldsymbol{p}\right]\left[\int_{\mathcal{H}^{2}} \int_{\mathcal{H}^{2}} S_{\mathrm{ir}}\left(\boldsymbol{\omega}_{i}, \omega_{o}\right) \mathrm{d} \omega_{i} \mathrm{~d} \omega_{o}\right]} \\
& \times S_{\text {ir }}\left(\omega_{i}, \omega_{o}\right) \int_{\mathcal{P}_{\text {all }}} T_{\text {ir }}\left(\boldsymbol{x}_{m}(\boldsymbol{p})\right) k_{\mathcal{P}_{\text {all }}}(\boldsymbol{p}) \mathrm{d} \boldsymbol{p} \\
& =\frac{T_{\mathrm{ir}}(\boldsymbol{x})}{\int_{\mathcal{P}_{\text {all }}} T_{\mathrm{ir}}\left(\boldsymbol{x}_{m}(\boldsymbol{p})\right) k \mathcal{P}_{\text {all }}(\boldsymbol{p}) \mathrm{d} \boldsymbol{p}} S_{\mathrm{ir}}\left(\boldsymbol{\omega}_{i}, \omega_{\boldsymbol{o}}\right) \int_{\mathcal{P}_{\text {all }}} T_{\mathrm{ir}}\left(\boldsymbol{x}_{m}(\boldsymbol{p})\right) k_{\mathcal{P}_{\text {all }}}(\boldsymbol{p}) \mathrm{d} \boldsymbol{p} \\
& =T_{\mathrm{ir}}(\boldsymbol{x}) S_{\mathrm{ir}}\left(\omega_{i}, \omega_{o}\right) \text {. }
\end{aligned}
$$

Therefore, the rank-1 factorization is consistent with our definition of $T_{\text {ir }}$ and $S_{\text {ir }}$. 